Article

\title{
Activeness and Loyalty Analysis in Event-Based Social Networks
}

\author{
Thanh Trinh *(D), Dingming Wu, Joshua Zhexue Huang and Muhammad Azhar $\mathbb{D}$ \\ College of Computer Science and Software Engineering, Shenzhen University, Shenzhen 518060, China; \\ dingming@szu.edu.cn (D.W.); zx.huang@szu.edu.cn (J.Z.H.); azhar@szu.edu.cn (M.A.) \\ * Correspondence: tthanh@szu.edu.cn
}

Received: 27 November 2019; Accepted: 16 January 2020; Published: 18 January 2020

\begin{abstract}
Event-based social networks (EBSNs) are widely used to create online social groups and organize offline events for users. Activeness and loyalty are crucial characteristics of these online social groups in terms of determining the growth or inactiveness of the social groups in a specific time frame. However, there is less research on these concepts to clarify the existence of groups in event-based social networks. In this paper, we study the problem of group activeness and user loyalty to provide a novel insight into online social networks. First, we analyze the structure of EBSNs and generate features from the crawled datasets. Second, we define the concepts of group activeness and user loyalty based on a series of time windows, and propose a method to measure the group activeness. In this proposed method, we first compute a ratio of a number of events between two consecutive time windows. We then develop an association matrix to assign the activeness label for each group after several consecutive time windows. Similarly, we measure the user loyalty in terms of attended events gathered in time windows and treat loyalty as a contributive feature of the group activeness. Finally, three well-known machine learning techniques are used to verify the activeness label and to generate features for each group. As a consequence, we also find a small group of features that are highly correlated and result in higher accuracy as compared to the whole features.
\end{abstract}

Keywords: social networks; EBSNs; activeness; loyalty

\section{Introduction}

Event-based social networks (EBSNs) [1], such as Meetup (www.meetup.com) or Douban (www.douban.com), have been rapidly developed as flexible platforms to create many types of online groups that users can join more conveniently than before. The groups often have specific themes, such as writing, cycling, and sports. In order to maintain the groups' active status, offline activities are often created monthly or even daily, for example, writing events and cycling events, and members are encouraged to attend. Having plentiful options, many users can join different online groups, and they may leave any group after a short period of time. In addition, groups with the same themes and events on similar topics are unavoidable in any kind of social network. Therefore, the shortage of loyal users makes some groups temporarily inactive or even permanently inactive. In contrast, other groups are still stably active and retain loyal users to attend the next events. These realities lead to a new research problem: activeness and loyalty analysis in event-based social networks. The research problem is critical for clarifying the existence and growth of these online groups. Thus, a detailed study on this research topic is the need of the current era.

Loyalty is considered as a fundamental concept that represents a relationship between clients and a company [2], as well as a relationship between users and their social groups [3]. Loyal users are considered a crucial factor in keeping their groups active. Therefore, the groups should provide useful services in order to gain new users and retain loyal ones. However, Palla et al. [4] and Jamali et al. [5] 
found that the tight relationships between users and their groups (small-sized or large-sized) determine the activeness level of the groups. Moreover, the relationships for the activeness of large groups continuously change over time. Hence, the concept of activeness in different social networks is different [6]. For example, active communities in following social networks, such as Instagram or Twitter, can be demonstrated as those who are able to follow many people, but they may be supporters of only one famous person. Similarly, in a citation network, a group of researchers is considered an active community if they refer to a hot research topic. However, the group becomes unstable due to the decrease in their interests in that research topic. The same thing also happens to a group of supporters who are loyal to a celebrity.

In this study, to address the research problem of activeness and loyalty in event-based social networks, we crawled data from the Meetup network in a given time frame. Our objective was to investigate the relationship between user loyalty and group activeness in diversified online groups in EBSNs. We mainly studied the activeness of groups in a series of consecutive time windows since the groups were created in the network. To reveal the characterization of the activeness of groups, we first defined sets of features derived from the crawled data. We then proposed a method to evaluate the activeness level of groups based on a ratio of a number of events between two consecutive time windows. Finally, we developed an association matrix to assign the activeness label for each group after the whole series of consecutive time windows.

Likewise, we measured the loyalty of users in several time windows. For this purpose, we utilized the change in the number of attended events of each user between two consecutive time windows to compute the loyalty of the user towards his/her group. The loyalty of the user was then treated as an essential feature in contributing to group activeness. We used three well-known machine learning techniques to validate the activeness label and the features of each group. We also found a group of correlated features that results in higher accuracy as compared to the features. According to our knowledge, no prior work has studied the problem of activeness and loyalty in EBSNs.

The remainder of this paper is organized as follows. Section 2 briefly reviews related work. Event-based social networks are analyzed, and sets of features are generated in Section 3. The measures of the activeness and loyalty are introduced in Section 4 . Section 5 presents the evaluation and analysis of the four real datasets. Conclusions are given in Section 6.

\section{Related Work}

Event-based social networks (EBSNs) had initially been investigated by Liu et al. [1]. Different types of recommendation problems were not only listed, but uniques and interesting characteristics of the networks were also analyzed in their work, such as information flows and locality structural groups. Various recommendation problems were studied in EBSNs [1,7-10]. However, the problem of activeness and loyalty in EBSNs still needs to be explored.

Detections of active communities and stable links in online social networks have been investigated by many researchers [6,11-14], and many techniques and methods have been proposed and developed [14-19].

The concept of activeness has been studied in several works [6,20-22]. In general, activeness presents the state of social processes within a specific time frame; thus, the notion of activeness is understood and bound by a specific period of time. Hence, the status of activeness can be different due to various periods of time: particularly active, stable, or inactive. Detecting active groups in a social network is valuable in understanding the essential characteristics of each group and development of the network. In [6], the authors studied community structures and proposed a framework to detect stable communities in directed social networks, such as Facebook and Twitter. They exploited the mutual links of all connections, and a Markov chain model was used to detect stable communities. Zhang et al. [13] studied a problem of stable link prediction field within a small group of Facebook users evolved in one selected month. They developed a new multiple linear regression model from a single model by using generated multi-variate features of links in that model. Zhang et al. [12] 
also adopted the multi-variate vector auto regression analysis approach to propose a new stable link detection method that improved the accuracy of stable link detection.

Quintane et al. [20] studied a problem of active social interactions and modeled them as important regularities in different time frames (short-time and long-time) to reveal distinct social processes. They used email datasets obtained from two separate teams in a project to carry out an empirical analysis. They concluded that the time frames were key to discovering the nature of the social processes, and the active process in a short-time period had an impact of social structure on individual, group, and organizational outcomes in a long-time period. In a different work [22], the authors defined a prominent actor as having active participation in one group and attending different groups regularly, and then proposed a new method to identify the actor in time-varying affiliation networks. Moreover, Patil et al. [11] studied a problem of predicting active online groups throughout two different datasets, i.e., an online game network (World of Warcraft) and a large co-authorship network (DBLP). They first examined individuals who had an influence on other users, and then proposed a model to predict the groups of those individuals that can remain stably active, and the groups can shrink over time.

The fundamental concept of loyalty was firstly used in marketing and business areas, where loyalty plays an important central role $[2,23]$. The notion of loyalty was used as a measure to understand customers' demands, so that companies can provide better products for their customers and thus gain more profits. Moreover, the development and maintenance of long-term relationships were treated as contributive factors in improving customer loyalty [24]. Gamboa et al. [25] used Facebook users to analyze the customer loyalty of the Zara company, and they created a list of key factors that determined the relationship between users and the company, i.e., trust, satisfaction, and perceived value factors. However, the lack of information in the virtual world caused the user loyalty to be unfaithful [26], and it became one of the major reasons of the inactiveness or collapse of many online groups. In another work, Hamilton et al. [27] studied the concept of loyalty in multiple online groups throughout the Reddit network; the concept was used for both the online community level and the user level. They found that loyal users within active communities have denser social interactions than those within inactive communities.

In our work, we focus on studying the activeness of groups and the loyalty of users. Loyalty is considered a valuable factor that contributes to the activeness of groups. Different from other social networks, event-based social networks do not have direct links between users.

\section{Data Collection and Feature Generation}

In this section, we first introduce the datasets and their structures crawled from the Meetup network. We then present the set of features generated from these obtained datasets.

\subsection{Meetup Data Collection}

We crawled data from the Meetup network, which is considered as one of the largest event-based social networks. In March 2019, Meetup had over 40 million users, and more than 10 thousand events were created every day in more than 180 countries through the Meetup network. The structure of EBSNs is demonstrated in Figure 1, which contains five main entities. Each event is created by one specific group and held in a particular venue at a given time. An event can be created by anyone in a group, and the event can be hosted by one or more event organizers. Each event has a list of RSVPs, which presents those who confirm YES or NO to take part in it. Users can join many groups, and each group is created by only one primary organizer.

Four crowded cities in three continents of the world were chosen in this study, i.e., London (LD), Sydney (SN), San Francisco (SF), and New York (NY). The data of these cities were crawled from the Meetup website, containing events only in groups created in 2014 and occurring from the beginning of 2014 to the end of 2016. The information of the users of those groups, who joined at that time, were also obtained. These crawled datasets are summarized in Table 1. 


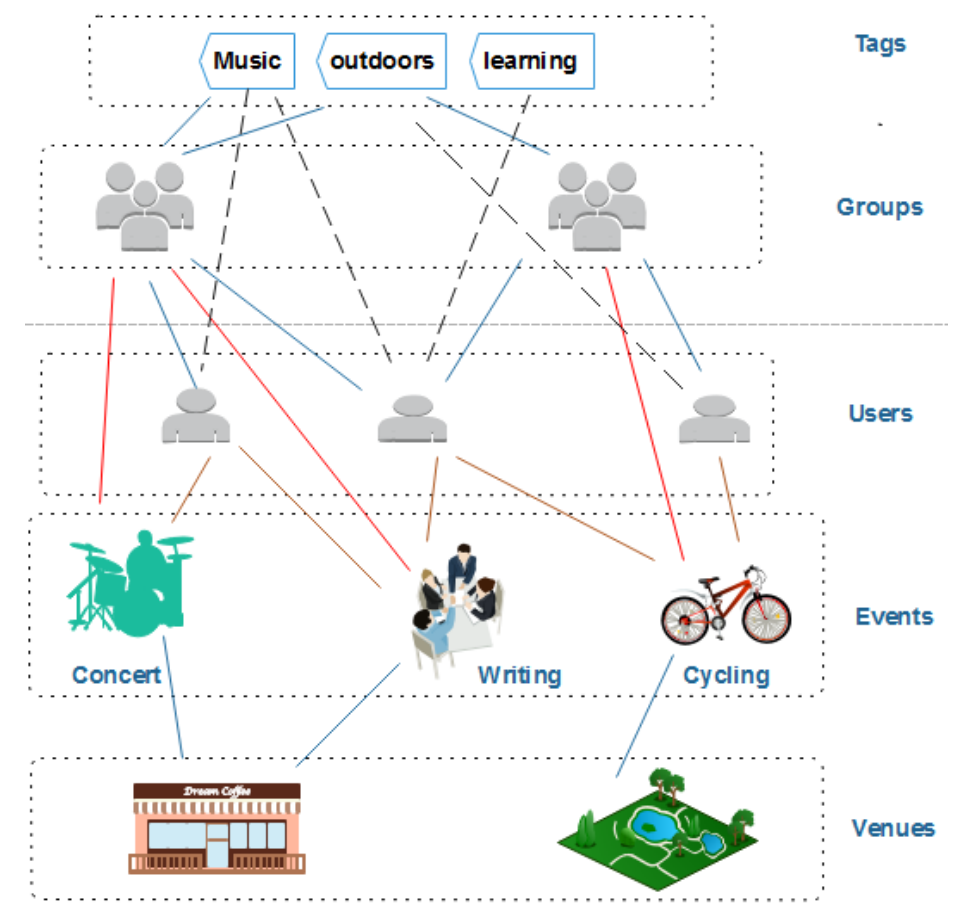

Figure 1. Example of an event-based social network (EBSN).

Table 1. Dataset statistics.

\begin{tabular}{cccccc}
\hline City & \#Groups & \#Events & \#Users & \#YES & \#NO \\
\hline New York & 1269 & 28,355 & 591,580 & 331,436 & 105,433 \\
San Francisco & 867 & 14,205 & 342,662 & 245,767 & 66,611 \\
London & 985 & 17,309 & 610,189 & 246,413 & 108,070 \\
Sydney & 297 & 5980 & 179,081 & 82,399 & 30,075 \\
\hline
\end{tabular}

We simply use the group category information gathered from the Meetup network. Table 2 shows 33 predefined categories; the type of each group is assigned in Table 2.

Table 2. Group categories.

\begin{tabular}{ccc}
\hline Alternative Lifestyle & Book Clubs & Career/Business \\
\hline Cars/Motorcycles & Community/Environment & Dancing \\
Education/Learning & Fashion/Beatuy & Fine Arts/Culture \\
Fitness & Food/Drink & Games \\
Health/Wellbeing & Hobbies & Language/Ethnic Identity \\
Lgbt & Movement/Politics & Movies/Films \\
Music & New Age/Spirituality & Outdoors/Adventure \\
Paranormal & Parents/Family & Pets/Animals \\
Photography & Religion/Beliefs & Sci-Fi/Fantasy \\
Singles & Socializing & Sports/Recreation \\
Support & Tech & Writing \\
\hline & &
\end{tabular}

\subsection{Features}

This section presents the features we use in the machine learning models. According to the structure of event-based social networks, we can generate a number of features from three main entities, i.e., group, event, and user entities. For further clarification of the presentation, we divide these features into three categories: group-based, event-based, and user-based features. Table 3 shows a list of generated features. 
Table 3. The features derived from datasets.

\begin{tabular}{|c|c|c|c|}
\hline Category & Feature & Description & Type \\
\hline \multirow{8}{*}{ Group-based } & CATEGORY & Corresponding category value & Integer \\
\hline & N_TOPICS & Number of topics in a group & Integer \\
\hline & N_USERS & Number of users in a group & Integer \\
\hline & RATING & Score average of group reviews & Double \\
\hline & YEAR & The year a group is created in & Integer \\
\hline & MONTH & The month a group is created in & Integer \\
\hline & DAY_OF_MONTH & The day a group is created on & Integer \\
\hline & WEEKDAY & The weekday a group is created on & Integer \\
\hline \multirow{13}{*}{ Event-based } & N_EVENTS & Number of events & Integer \\
\hline & RSVPs & Number of all RSVPS & Integer \\
\hline & Y_RSVPs & Number of all RSVPS only with YES & Integer \\
\hline & N_RSVPs & Number of all RSVPS only with NO & Integer \\
\hline & AVERĀGE_RSVPs & Average of all RSVPSs & Double \\
\hline & SD_RSVPs & Standard deviation of all RSVPs & Integer \\
\hline & AVERAGE_Y_RSVPs & Average of RSVPS only with YES & Integer \\
\hline & SD_Y_RSVPS & Standard deviation of all RSVPS only with YES & Integer \\
\hline & AVERAGE_N_RSVPs & Average of RSVPS only with NO & Integer \\
\hline & SD_N_RSV̄Ps & Standard deviation of all RSVPS only with NO & Integer \\
\hline & AVERAGE_DAY & Average days between two consecutive events & Double \\
\hline & SD_DAY & Standard deviation of numbers of days between two consecutive events & Double \\
\hline & N_EVENT_ORGANIZER & Number of events that has organizers & Double \\
\hline \multirow[t]{4}{*}{ User-based } & N_ORGANIZER & Number of organizers in the group & Integer \\
\hline & N_ATTENDEES & Number of users who confirm at least one YES & Integer \\
\hline & BIO & Number of users who have a biography & Integer \\
\hline & ADDRESS & Number of users who have address information & Integer \\
\hline
\end{tabular}

Group-based features. These features are derived from the basic information of each group. The characteristics of these features are discussed in detail as follows.

The CATEGORY feature is obtained from Table 2 for each group, and each group has only one category value. Different to the CATEGORY feature, the N_TOPICS feature denotes a number of topics that the group is interested in. Those topics are selected by the primary group organizer, and they can be manifested by a set of tags, as shown in Figure 1. The number of users in each group is considered an important feature, denoted by N_USERS. This feature N_USERS reflects the growth of groups. In addition to utilize the crawled data, the rating level and creation time of each group are also taken into account. RATING is a score of the average of group reviews, and creation time provides information of the time that a group was created in YEAR, MONTH,DAY_OF_MONTH, and WEEKDAY.

Event-based features. These features represent the information of all events created by each group, and reflect the diversity of activities in the group. We create the set of features from this information as follows.

The number of events $N \_E V E N T S$ in each group is derived as a crucial feature to evaluate the growth of the group. Each event has a set of RSVPs that present those who make a confirmation of whether to attend the event or not. The RSVPs feature presents a sum of all sets of RSVPs in all events created by each group. Furthermore, features $Y_{-} R S V P S$ and $N_{-} R S V P S$ that are obtained from the RSVPS feature present the total number of RSVPS with $Y E S$ and that with $N O$, respectively. The AVERAGE_RSVPS and $S D_{-} R S V P S$ features represent the average of all sets of RSVPS and the standard deviation of all sets of RSVPS in the group. Similarly, the $A V E R A G E_{-} Y_{-} R S V P S, S D_{-} Y_{-} R S V P S, A V E R A G E_{-} N_{-} R S V P S$, and $S D_{-} N_{-} R S V P S$ features are generated from $Y \_R S V P S$ and $N_{-} R S V P$ s. These features also reflect the loyalty of users who take part in events. The $A V E R A G E \_D A Y$ and $S D \_D A Y$ features present the average of days between two consecutive events and the standard deviation of them for each group. These two features characterize the activity of the group; for example, events are held weekly or monthly. The number of events that have organizers is represented in the N_EVENT_ORGANIZER feature.

User-based features. These features reveal the properties of all users in each group. This set also reflects the activities of active users in the group. 
The number of organizers, who manage events in the group, is denoted by the N_ORGANIZER feature. The number of users who attend at least one event in each group is defined in the N_ATTENDEES feature, which describes the density of active members in the group. BIO and $A D D R E S S$ features are obtained from the users who have biography information and those who have addresses in the crawled datasets, respectively.

\section{Methodology}

In this section, we propose novel methods to measure the activeness of groups and the loyalty of users. To predict the activeness of groups, three well-known machine-learning techniques are used, and results have been shown to validate our methods.

\subsection{Method to Measure Group Activeness}

In the multi-online groups platform like Meetup, one key characteristic that emerges to clarify the active or inactive status of each group is the number of events in a specific period of time. Because of different types of groups, the numbers of events of groups are different in the same time period. For example, some groups have events daily or weekly, while others arrange only one event in a month or even in three months. Hence, the change in the number of events in each group between two consecutive time intervals is adopted to evaluate whether the group is active or not. In other words, the change in the number of events is used to specify three levels of group activeness, i.e., active, stable, and inactive, because this feature strongly reflects the group activeness than any other features. Therefore, we define the concept of group activeness in EBSNs based on the numbers of events that are created in different consecutive time windows. Table 4 lists the notations used in this paper.

Table 4. Notations.

\begin{tabular}{ccc}
\hline$G$ & Group $G$ & \\
$T^{i}$ & The $i$ th time window of group $G$ & \\
$E$ & Event & \\
$N^{i}$ & The number of events created by group $G$ in $T^{i}$ & Integer \\
$R$ & Ratio of $N^{i}$ and $N^{i-1}$ & Double \\
$\lambda$ & The measure of the loyalty of a user & Double \\
\hline
\end{tabular}

To measure activeness, we propose a method that is used to label the activeness level of each group after a set of consecutive time windows. The proposed method consists of two steps. Inspired by calculating the journal impact factor from Clarivate Analytics organization (https:/ / clarivate.com/), first we compute the ratio on the numbers of events in two consecutive time windows and assign each group with an activeness label. After that, we develop an association label matrix to reassign a new label for each group after several consecutive time windows. We do not consider more consecutive windows in deciding group activeness because different groups have different histories. It will complicate the matter if multiple time windows are used to compute the activeness of different groups. The process of this method is expressed in the following details.

Activeness labels. Given a group $G$ and a given set of consecutive time windows, $\left\{T^{1}, \ldots, T^{n}\right\}$, in which each $T^{i}$ has a corresponding number of events $N^{i}$ created by $G$, as illustrated in Figure 2, we first calculate the ratio of events $R$ between $T^{i}$ and $T^{i-1}$ as the following equation:

$$
R=\frac{N^{i}}{N^{i-1}}
$$

where $N^{i}$ and $N^{i-1}$ are the numbers of events of the group $G$ in $T^{i}$ and $T^{i-1}$, respectively. Note that, if both $N^{i}$ and $N^{i-1}$ are equal to 0 , the value of $R$ is assigned to 0 . On the other hand, if $N^{i}>0$ and $N^{i-1}=0$, then the value of $R$ is assigned by $N^{i}$. 


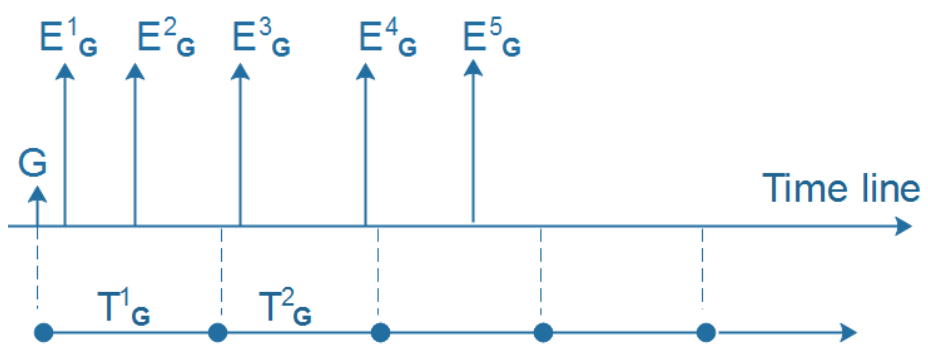

Figure 2. Example of the time frame of group $G$.

The label of the group $G$ in the period of $T^{i-1, i}$ is then defined as follows:

$$
G_{L}= \begin{cases}A: \text { Active, } & \text { if } R \geq 1 \\ S: \text { Stable, } & \text { if } 0.5 \leq R<1 \\ I: \text { Inactive, } & \text { if } R<0.5\end{cases}
$$

where $R$ is the ratio computed in Equation (1), and $L$ denotes the labels of the group.

Finally, we develop the following label matrix to assign an activeness label for the group $G$ after the whole set of time windows $T^{1, \ldots, n}$.

$$
\text { label matrix }=S^{n-1, n} A^{n-1, n}\left[\begin{array}{ccc}
I^{1, \ldots, n-1} & S^{1, \ldots, n-1} & A^{1, \ldots, n-1} \\
I & I & S \\
S & S & A \\
S & A & A
\end{array}\right]
$$

Specifically, if $S^{1, . . n-1}$ is the activeness label of $G$ in the period of $T^{1, \ldots, n-1}$, and $A^{n-1, n}$ is the label in the period of $T^{n-1, n}$. The activeness label of $G$ in the whole $T^{1, \ldots, n}$ is assigned by $A$.

The following example describes the process of assigning the activeness label for groups.

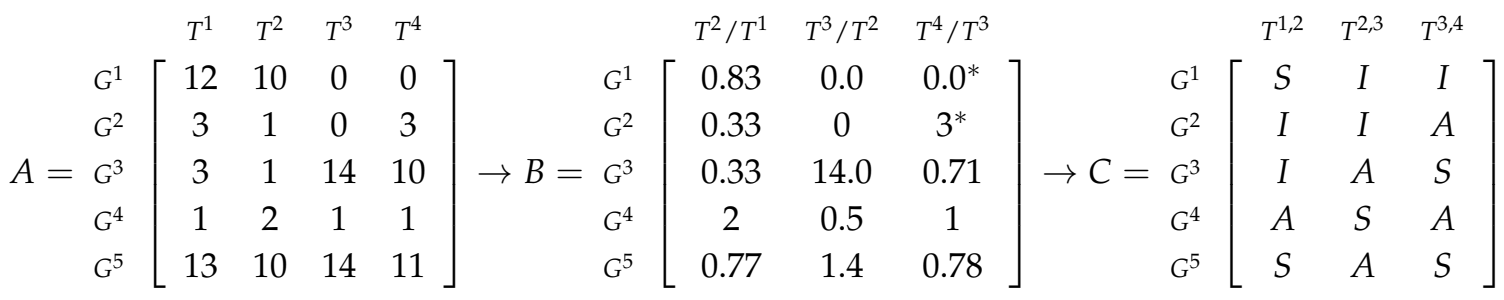

$$
\begin{aligned}
& \left.\rightarrow D=\begin{array}{l}
G^{1} \\
G^{2} \\
G^{4} \\
G^{5}
\end{array}\left[\begin{array}{cc}
T^{1,2,3} & T^{3,4} \\
I & I \\
S & A \\
A & A \\
A & S
\end{array}\right] \rightarrow \text { Activeness label }=G^{3}\left[\begin{array}{c}
G^{1} \\
G^{2} \\
G^{4}
\end{array}\right] \begin{array}{c}
I \\
S \\
S \\
A \\
A
\end{array}\right]
\end{aligned}
$$

Example of activeness label. The matrix $A$ describes the numbers of events of five groups $\left(G^{1}, \ldots, G^{5}\right)$ in four consecutive time windows. First, the ratios of the numbers of events between two consecutive time windows for each group are calculated as shown in the matrix $B$. Note that the values in the matrix $B$ with a marked $*$ are also assigned based on Equation (1). Then, the matrix $C$ illustrates the activeness label of each group in the two consecutive time windows, the labels are assigned based on Equation (2). To label these groups in the period of the first three time windows, i.e., $T^{1}, T^{2}$, and $T^{3}$, we select the first two columns, i.e., $T^{1,2}$ and $T^{2,3}$, in the matrix $C$. After that, we use the label matrix to assign a label to each corresponding group for the period of $T^{1,2,3}$. The results of the five groups for 
the period of $T^{1,2,3}$ are shown in the first column in the matrix $D$, and the second column $T^{3,4}$ in $D$ is still the activeness label obtained from the matrix $C$. Finally, we use the label matrix for the matrix $D$ to achieve the activeness label for the five groups after the period of $T^{1,2,3,4}$, as illustrated in the activeness label matrix.

\subsection{Method to Measure User Loyalty}

Similarly, we use the time windows to define the loyalty of users towards their group. The concept of user loyalty is defined based on participation, which is considered as a strong view for clarifying loyal users. To measure the loyalty of a user $u$ in his/her group $G$, we first obtain attended events by user $u$ in one $T^{i}$. We then calculate $P^{i}$, which is the ratio of attended events of $u$ to all events of $G$ created in $T^{i}$. Finally, we measure the loyalty of user $u$ after several consecutive time windows as follows.

$$
\lambda=\frac{\sum_{i=1}^{n} P^{i}}{n}
$$

where $n$ is the number of consecutive time windows. The value of $\lambda$ is from 0 to 1 . If the value is close to 1 , the user $u$ is loyal to the group $G$. Otherwise, if the value is near to 0 , the user $u$ is the disloyal user.

We use a given threshold $\tau$ to differentiate loyal users and disloyal users within $n$ consecutive time windows. If $\lambda \geqslant \tau$, which means $u$ is considered the loyal user; for example, $u$ is the loyal user of $G$ within $T^{1,2}$. The number of loyal users of $G$ is denoted by the LOYAL_USERS feature.

\subsection{Prediction Techniques}

As discussed above, one group has a set of generated features and a corresponding activeness label. The set of these features consists of user-based features, group-based features, event-based features, and the LOYAL_USERS feature. To evaluate the set of generated features and the activeness label for each group, we adopt supervised classifiers. The random forest (RF) method [28,29] can predict high accuracy even with a set of weak features. The decision tree method [30] can select a group of strong features to construct a prediction tree. Moreover, these methods are nonparametric ones. The support vector machine (SVM) [31] is another well-known method that is very useful for a group of features that are highly correlated. Therefore, we selected these three methods to evaluate the activeness label of each group by using the set of features of the group. The process of evaluation is described in Section 5.4 .

\section{Evaluation and Analysis}

The datasets that we used in our experiments have been described in Section 3. They contain groups that were created in the year 2014, and all event datasets of each group that were created by the end of the year 2016 were obtained. All users of each group were also selected in that time.

Figure 3 represents the category type distribution in the online groups created in the four cities in the year 2014. We can observe that a majority of groups belongs to the tech and career/business category type.

\subsection{Time Window}

We evaluate the activeness of groups by dividing a time period of each group in several time windows from its creation date. To select a sliding time window $T$ in the lifetime, all events that have been created by each group are obtained in order to compute the average time between two consecutive events in the group. We plot the distribution of average days between two consecutive events created in each group for all datasets, as illustrated in Figure 4. It is observed that the majority of the average time periods are less than 90 days. Therefore, we set $T$ with the interval of 90 days to evaluate the activeness of all groups in the four cities. Thus, we create a series of eight consecutive Ts for all groups in all datasets, the series is equivalent to the period of 2 years. 

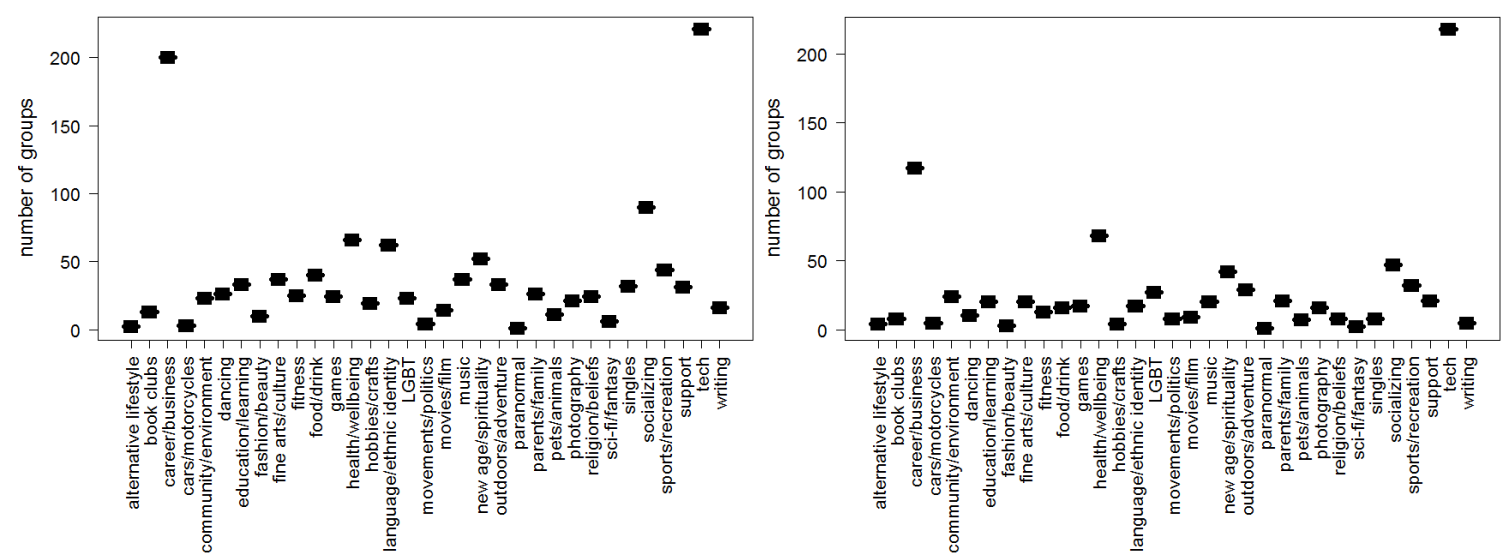

(a) New York

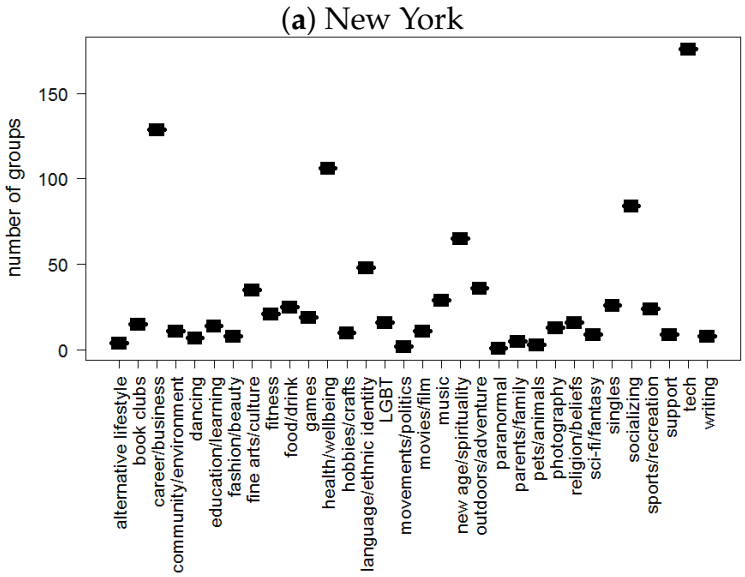

(c) London

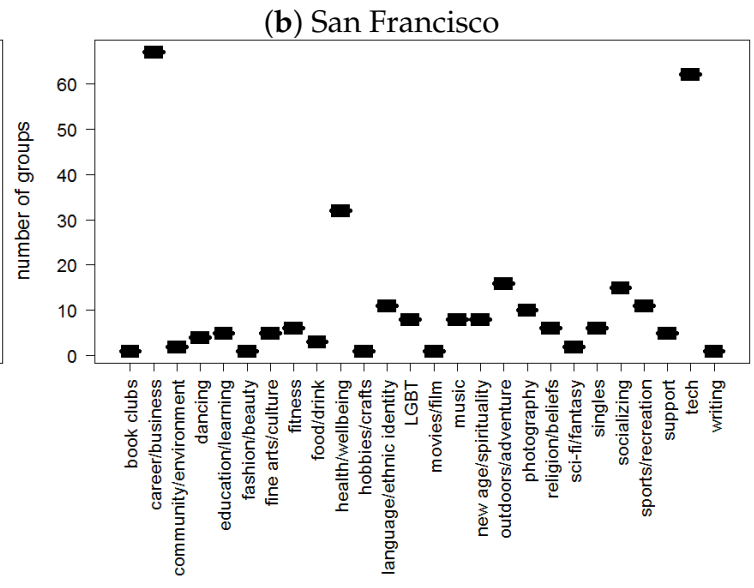

(d) Sydney

Figure 3. Category type distribution in groups created in four cities in the year 2014.

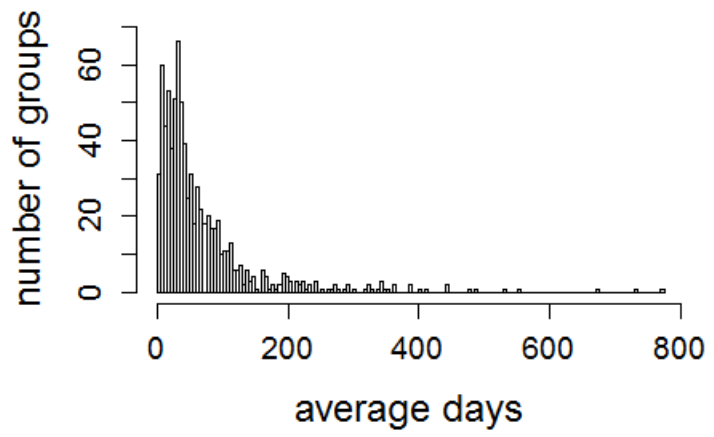

(a) New York

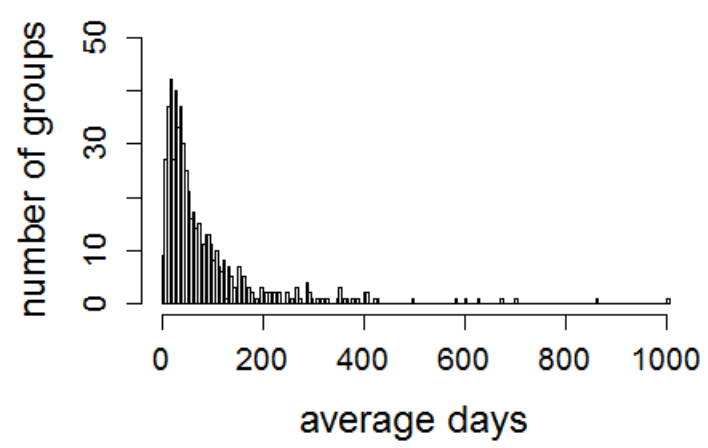

(b) San Francisco

Figure 4. Cont. 


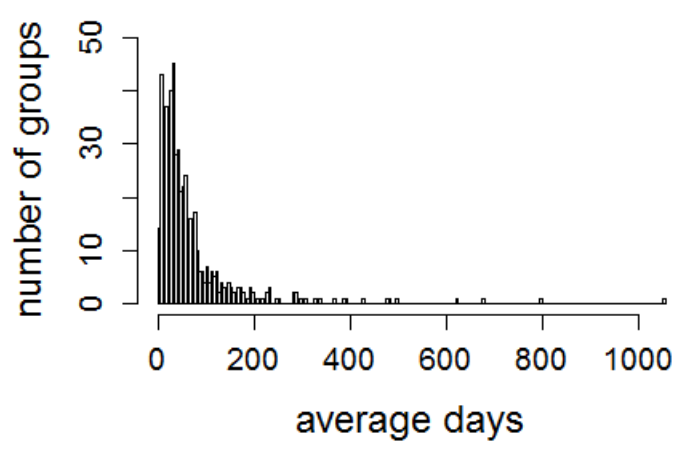

(c) London

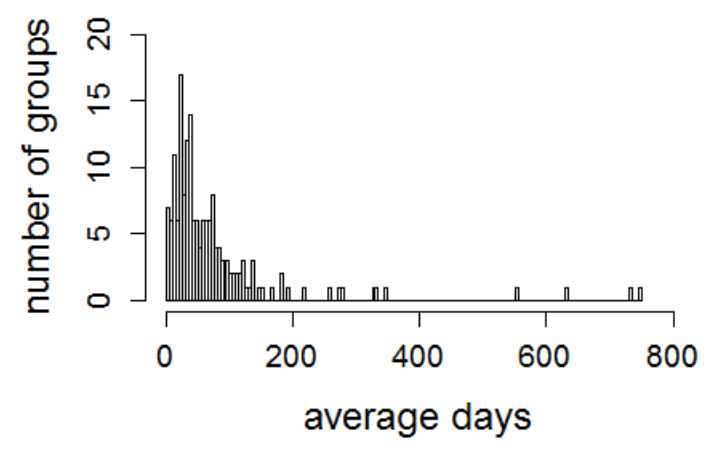

(d) Sydney

Figure 4. Distribution of average days between two consecutive events in each group.

Figure 5 demonstrates the time windows $T$ of group $G^{1}$ and group $G^{2}$. The creation time of these two groups is different; therefore, the starting point and ending point of each $T$ for each group are different. Each group has a number of events in each $T$.

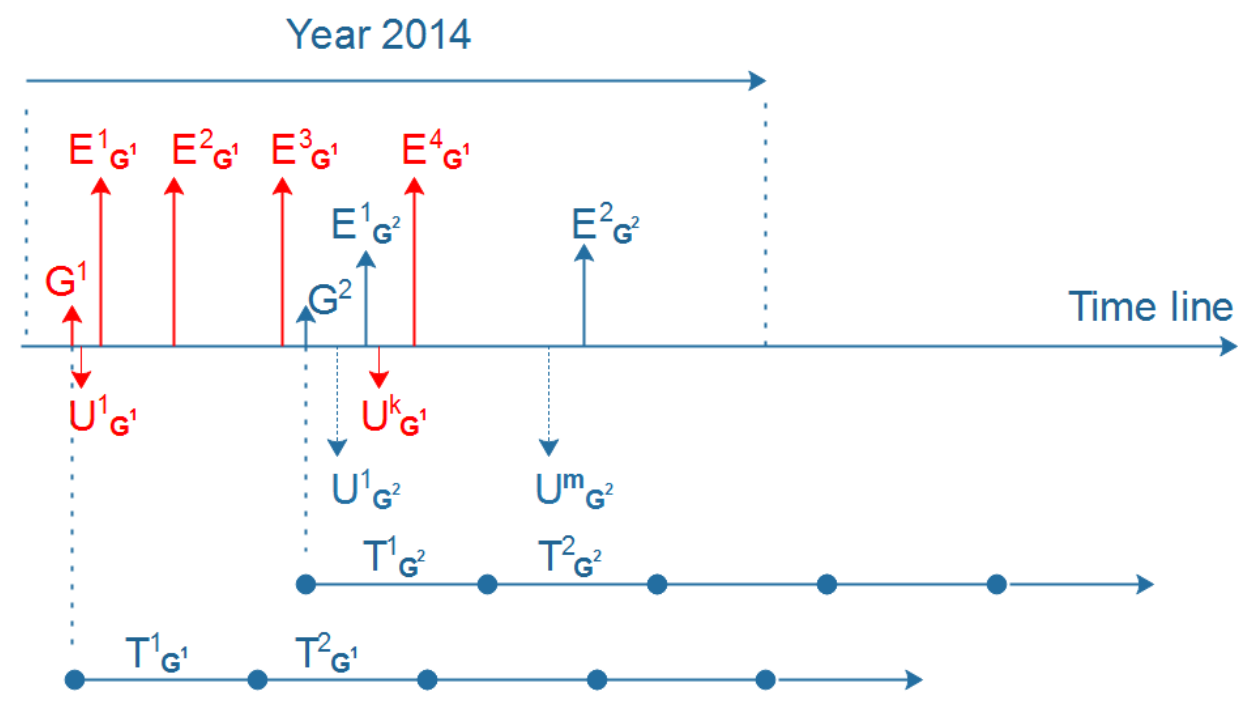

Figure 5. Example of the time window $T$ in $G^{1}$ and $G^{2}$.

\subsection{Group Activeness Label}

For each group $G$ in all datasets, we created a corresponding series of 8 time windows, i.e., $T_{G}^{1, \ldots, 8}$. For each $T_{G}^{i}$, we collect all events created in $T_{G}^{i}$ to assign the activeness label for the group. However, we only evaluate groups that create at least one event in the first time window $T^{1}$. If a group does not have any event in $T^{1}$, the group will be removed in the process of evaluation. Otherwise, the group will be taken into the evaluation of activeness.

Figures 6-9 present the numbers of groups and the total numbers of events created by those groups in each $T^{i}$ of the four cities. These four figures also describe the changes in the numbers of events created by each group between two consecutive $T$. In these figures, each $X$ denotes a group. The straight line represents the points where the numbers of events in two consecutive windows are equal. In general, the numbers of those groups steadily decrease in the 2-year lifetime for all 4 cities. However, the numbers of events that are held in each $T$ fluctuate. This means, in a time period $T^{i}$, the number of events created by various groups sharply increased or decreased, as compared to $T^{i-1}$. 


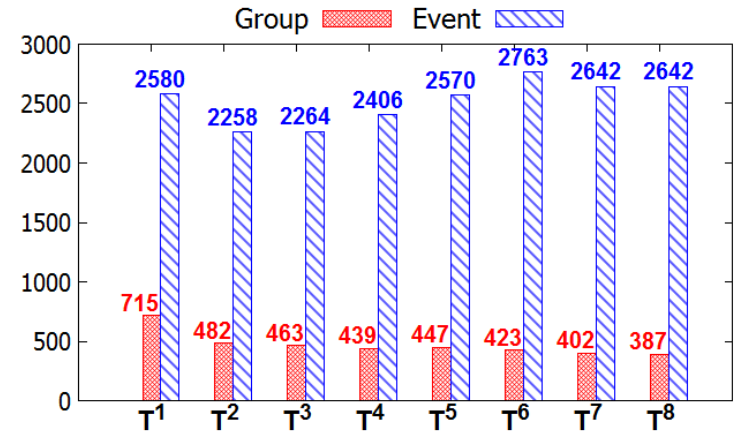

(a) Number of groups and events in each $T$.

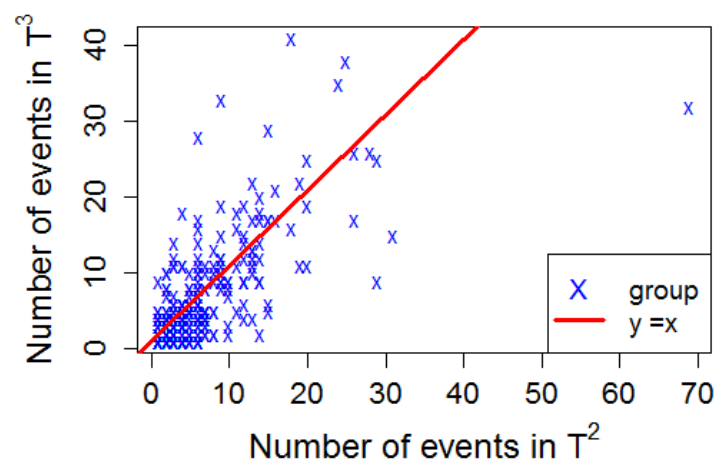

(c) Events created by each group in $T^{2}$ and $T^{3}$.

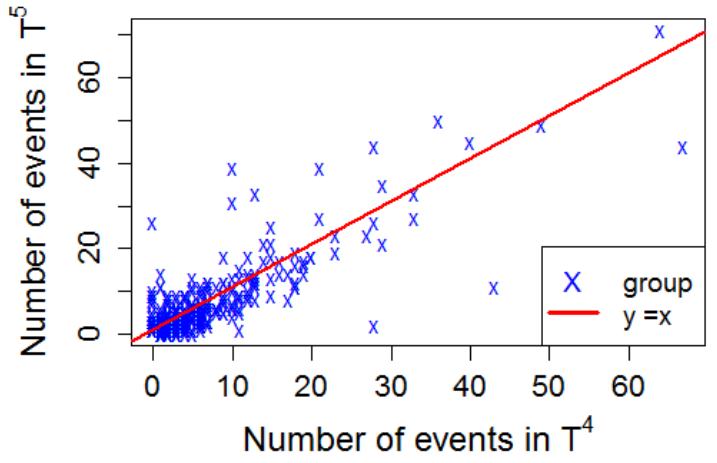

(e) Events created by each group in $T^{4}$ and $T^{5}$.

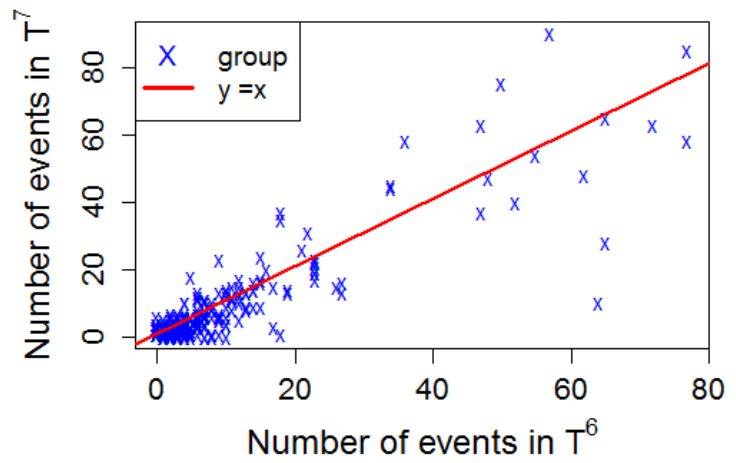

(g) Events created by each group in $T^{6}$ and $T^{7}$.

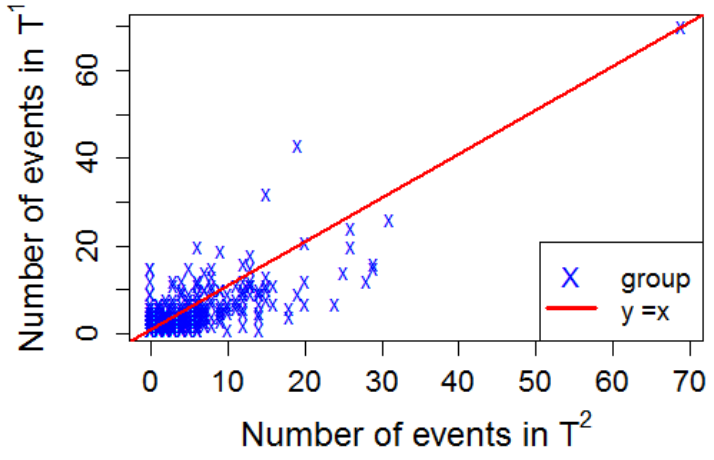

(b) Events created by each group in $T^{1}$ and $T^{2}$.

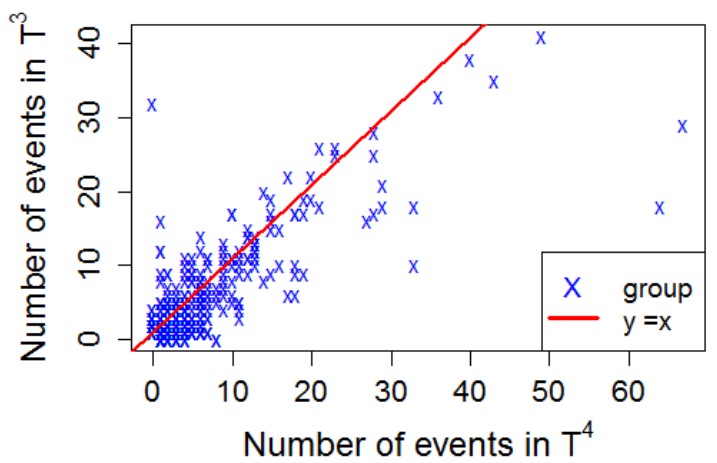

(d) Events created by each group in $T^{3}$ and $T^{4}$.

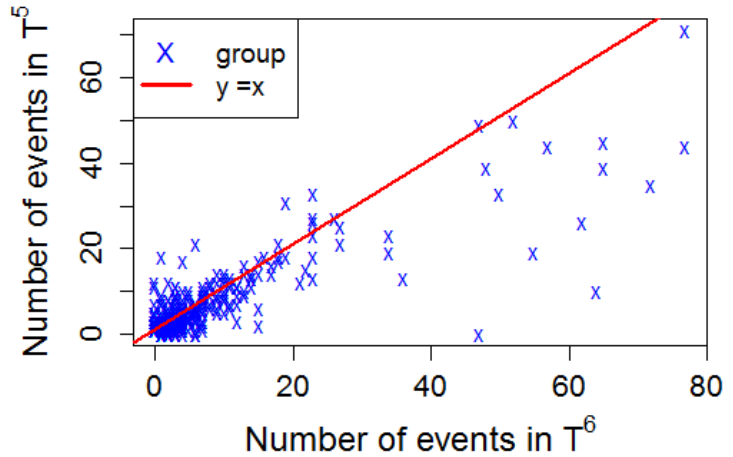

(f) Events created by each group in $T^{5}$ and $T^{6}$.

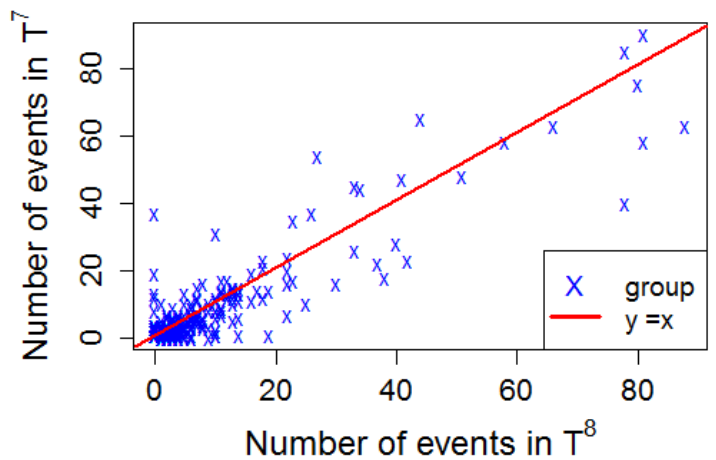

(h) Events created by each group in $T^{7}$ and $T^{8}$.

Figure 6. New York-The numbers of groups and events in 8 time windows. 


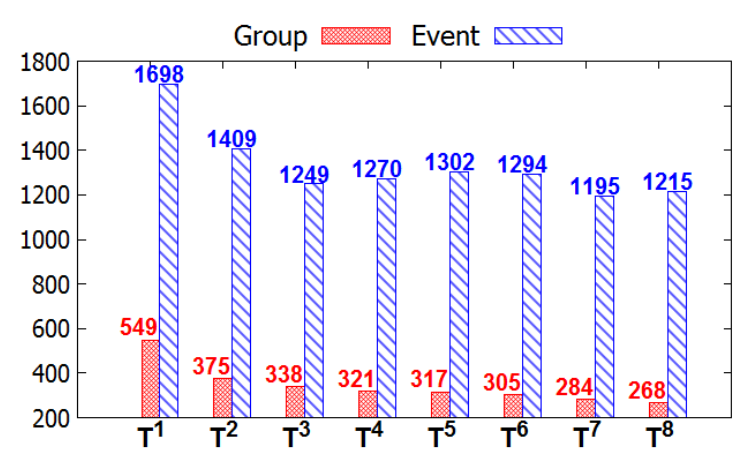

(a) Number of groups and events in each $T$.

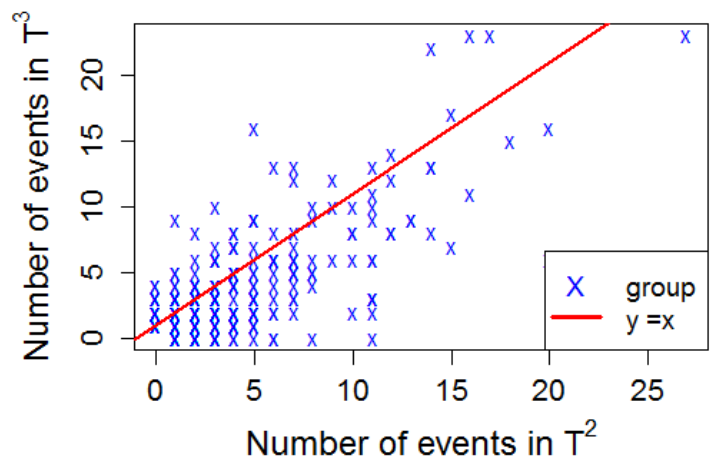

(c) Events created by each group in $T^{2}$ and $T^{3}$.

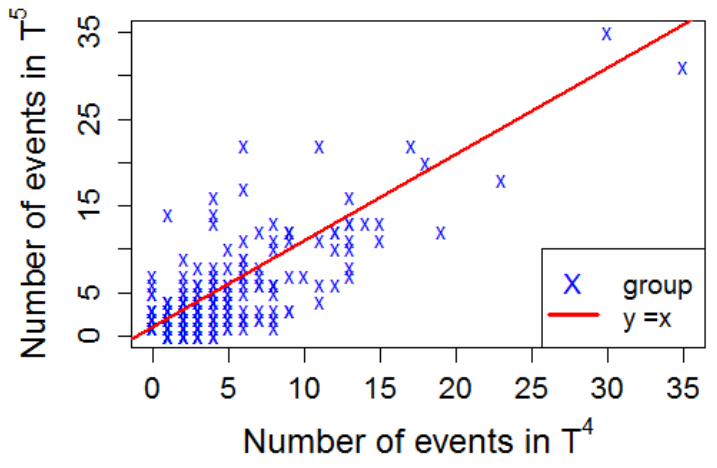

(e) Events created by each group in $T^{4}$ and $T^{5}$.

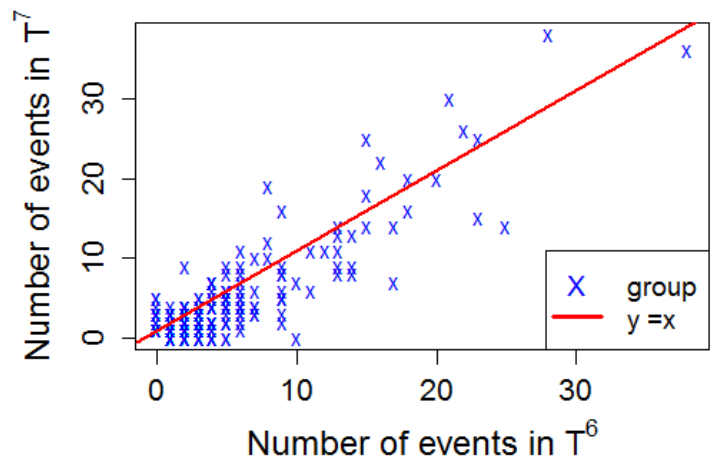

(g) Events created by each group in $T^{6}$ and $T^{7}$.

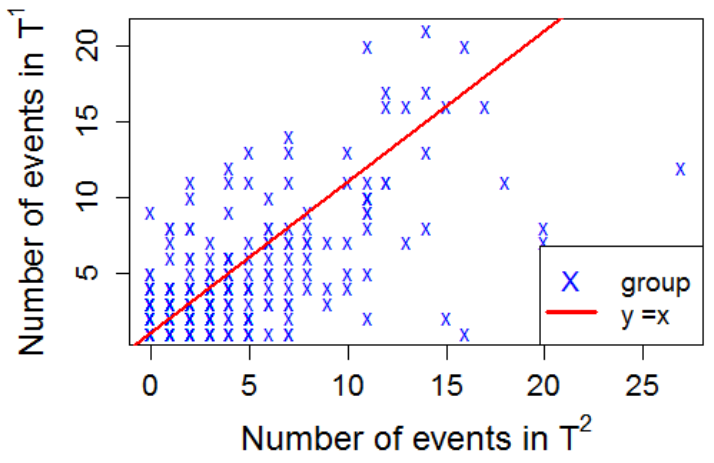

(b) Events created by each group in $T^{1}$ and $T^{2}$.

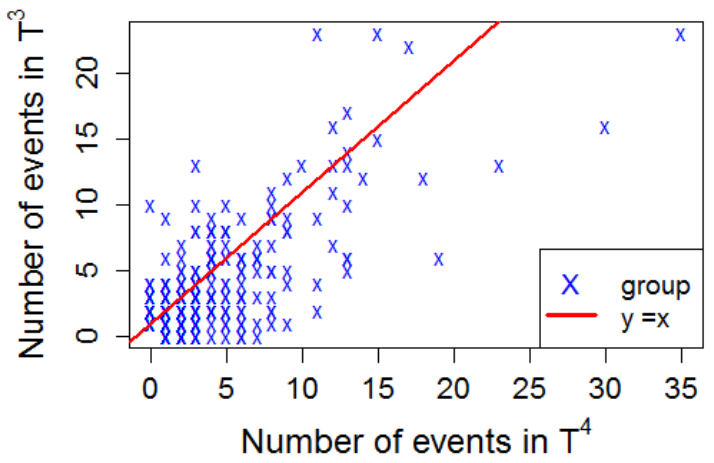

(d) Events created by each group in $T^{3}$ and $T^{4}$.

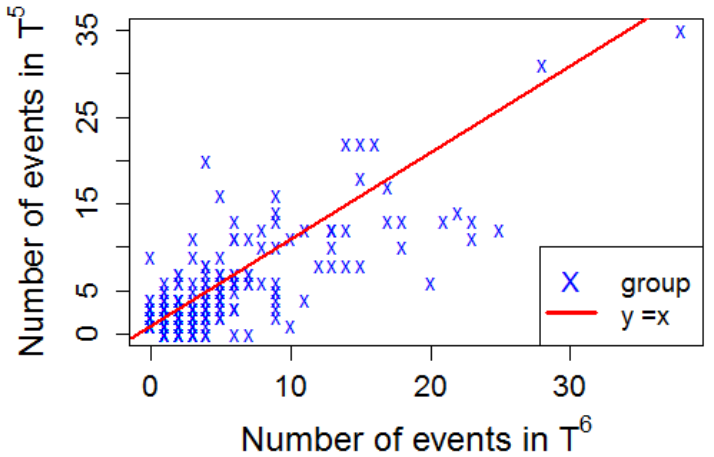

(f) Events created by each group in $T^{5}$ and $T^{6}$.

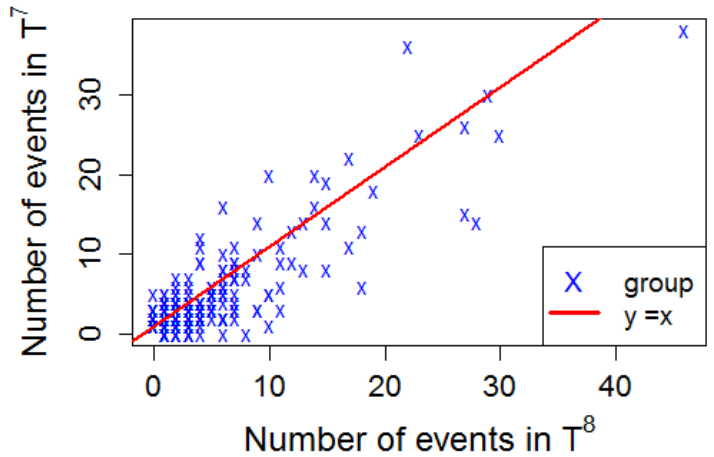

(h) Events created by each group in $T^{7}$ and $T^{8}$.

Figure 7. San Francisco-The numbers of groups and events in 8 time windows. 


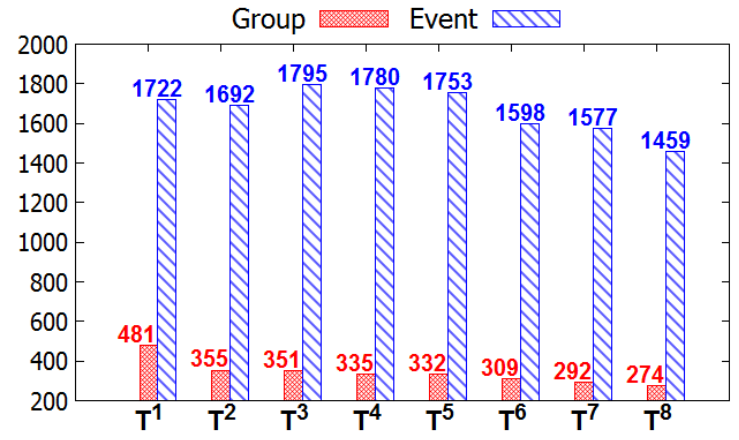

(a) Number of groups and events in each $T$.

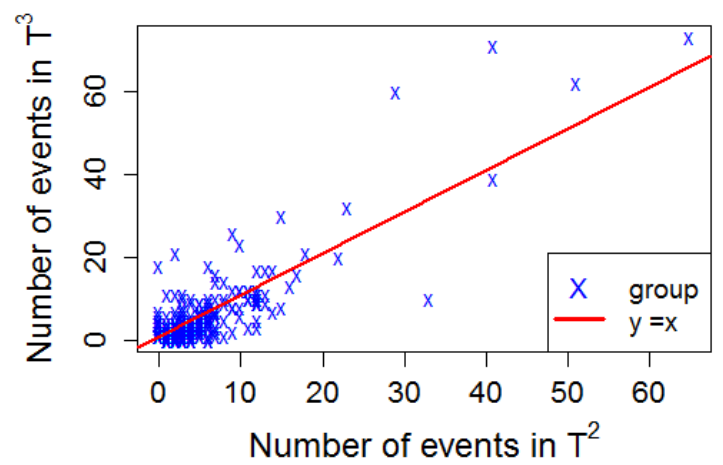

(c) Events created by each group in $T^{2}$ and $T^{3}$.

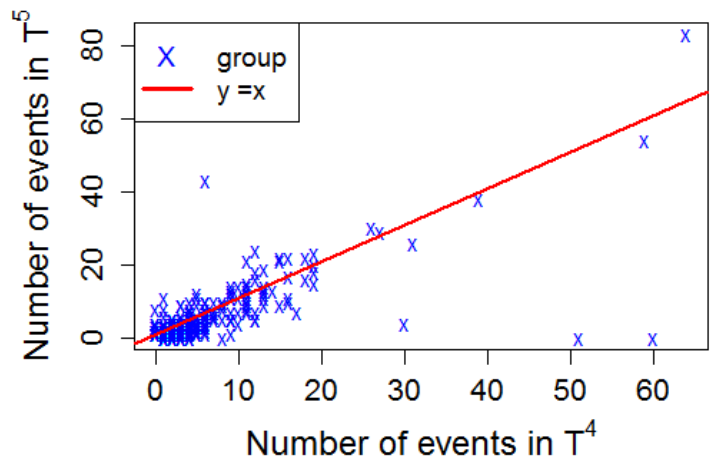

(e) Events created by each group in $T^{4}$ and $T^{5}$.

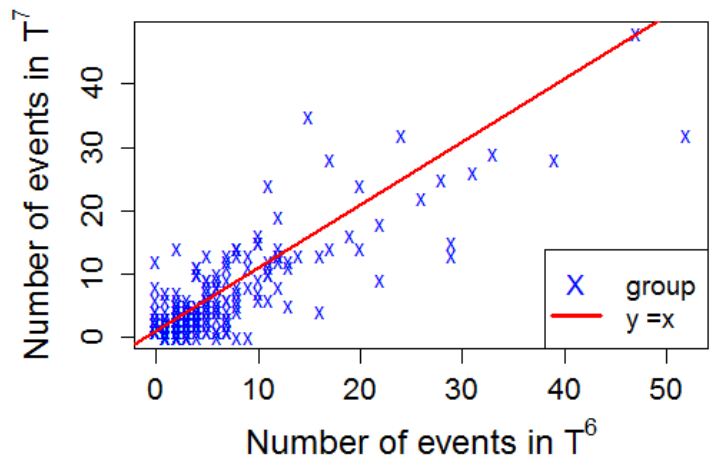

(g) Events created by each group in $T^{6}$ and $T^{7}$.

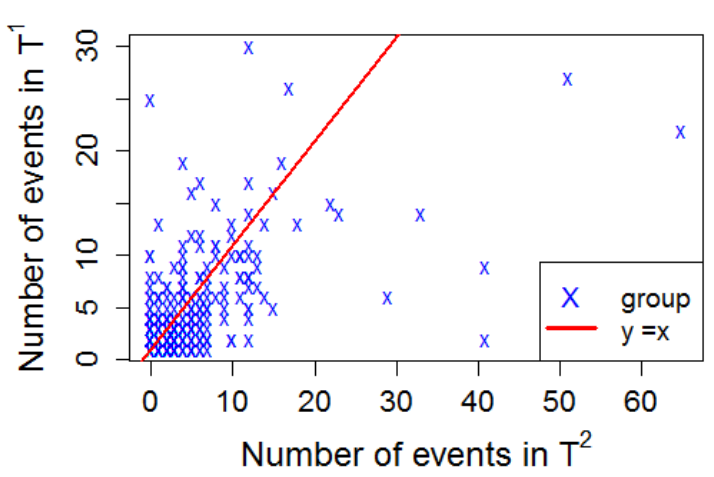

(b) Events created by each group in $T^{1}$ and $T^{2}$.

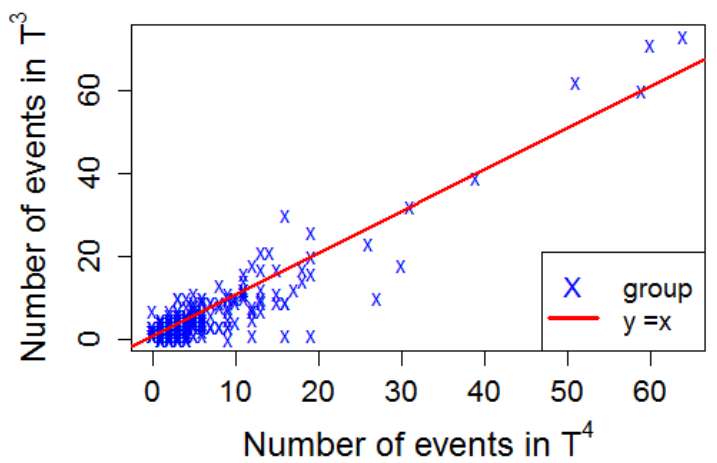

(d) Events created by each group in $T^{3}$ and $T^{4}$.

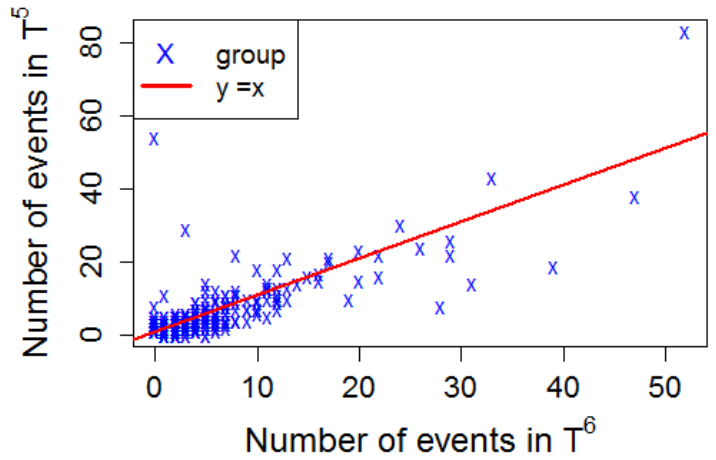

(f) Events created by each group in $T^{5}$ and $T^{6}$.

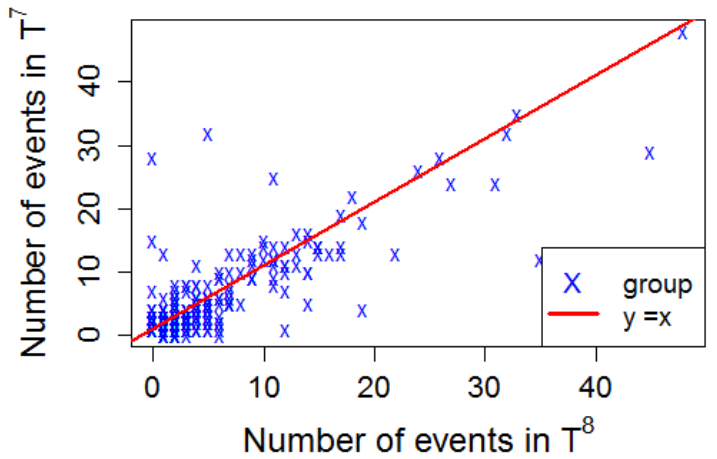

(h) Events created by each group in $T^{7}$ and $T^{8}$.

Figure 8. London-The numbers of groups and events in 8 time windows. 


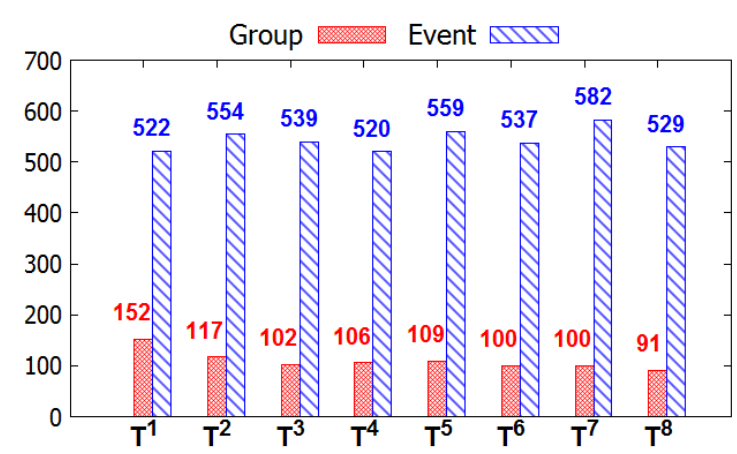

(a) Numbers of groups and events in each $T$.

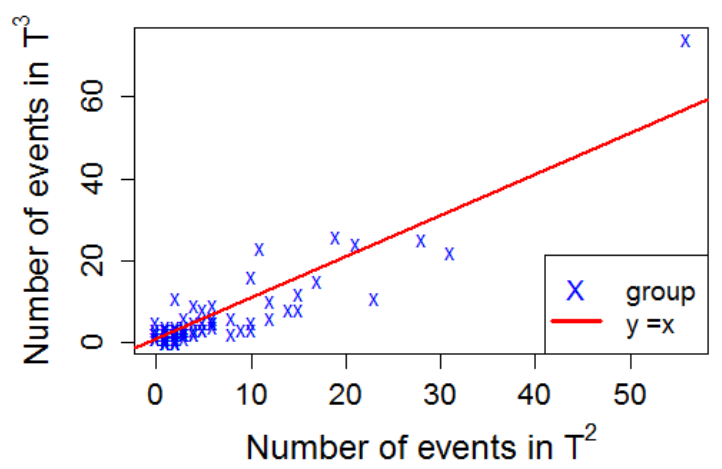

(c) Events created by each group in $T^{2}$ and $T^{3}$.

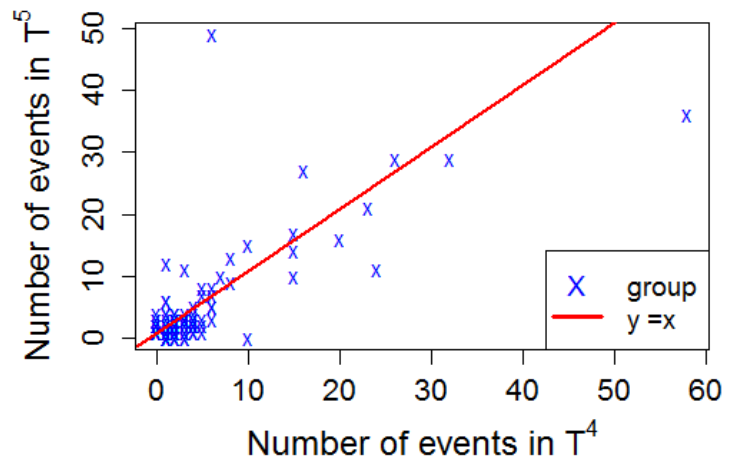

(e) Events created by each group in $T^{4}$ and $T^{5}$.

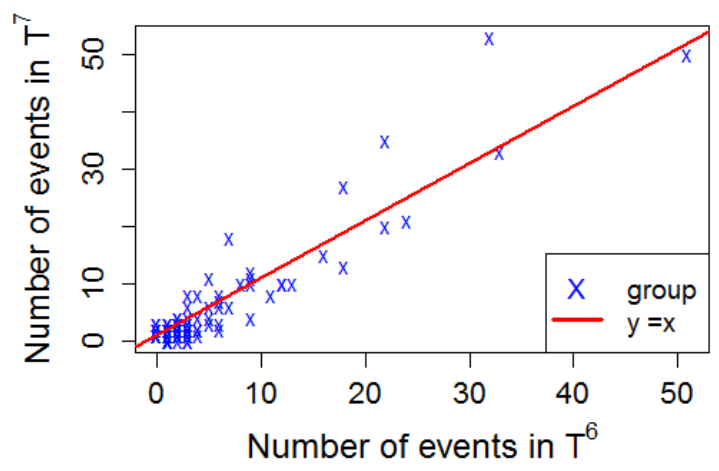

(g) Events created by each group in $T^{6}$ and $T^{7}$.

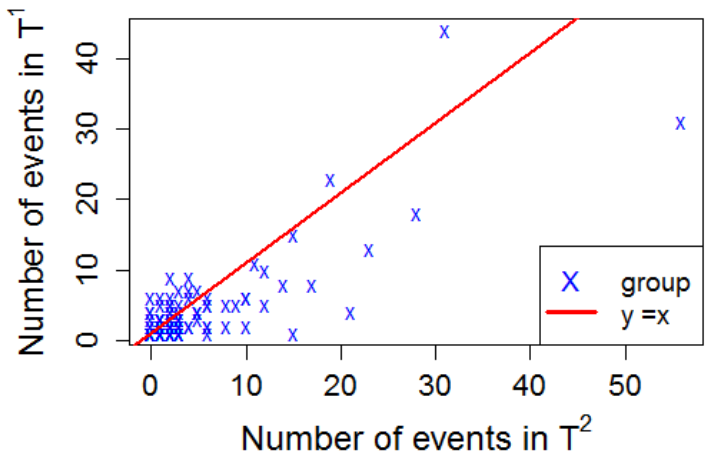

(b) Events created by each group in $T^{1}$ and $T^{2}$.

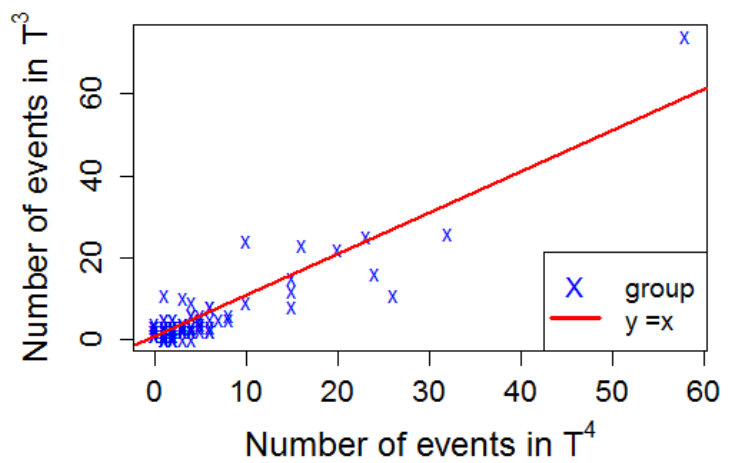

(d) Events created by each group in $T^{3}$ and $T^{4}$.

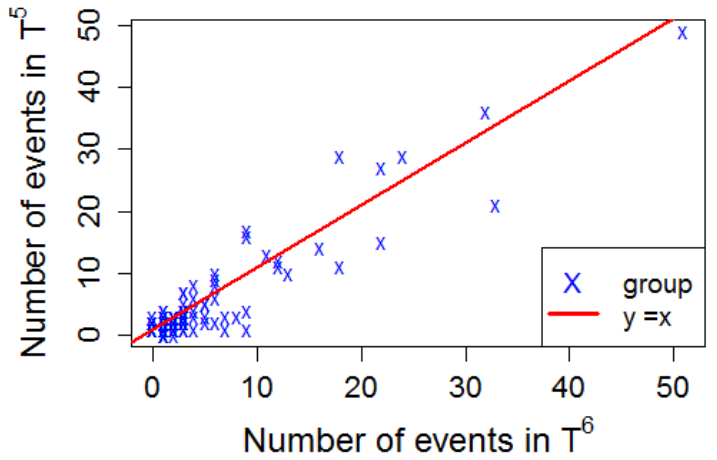

(f) Events created by each group in $T^{5}$ and $T^{6}$.

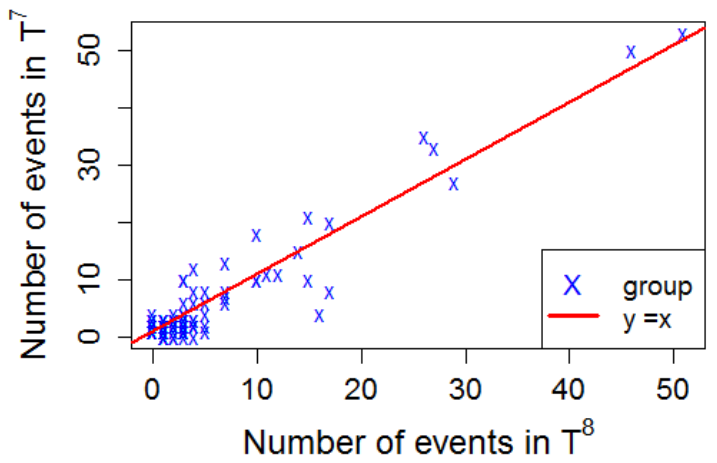

(h) Events created by each group in $T^{7}$ and $T^{8}$.

Figure 9. Sydney-The numbers of groups and events in 8 time windows. 
To evaluate the activeness of group $G$ in terms of the number of events, we first collect all events of $G$ in each $T$. We then use Equation (1) to compute $R$, the ratio of the numbers of events between two consecutive $T$, for example $T^{3}$ and $T^{4}$. We use Equation (2) to assign the activeness label of $G$ between $T^{3}$ and $T^{4}$. Finally, the association label matrix is used to assign the activeness label for each group after several consecutive time windows.

Table 5 shows the activeness labels of all groups in the four cities during several consecutive time windows where the first column Total in Table 5a-d describes the numbers of groups that have at least one event in $T^{1}$. We can observe that the numbers of groups in the Active label achieved the highest value after one year in San Francisco and London, while those numbers in New York and Sydney achieved the highest value after one year and three months. After that, the numbers steadily deceased. Moreover, the numbers of groups with a Stable label have also shown a similar trend. Therefore, we can conclude that we can predict groups that are inactive or active in online social networks after one year.

Table 5. The distribution of numbers of groups in three different activeness labels after several time windows in the four cities.

\begin{tabular}{lcccclcccc}
\hline & Total & Inactive & Stable & Active & & Total & Inactive & Stable & Active \\
\hline$T^{1}$ & 715 & & & & $T^{1}$ & 549 & & & \\
$T^{1,2}$ & 715 & 286 & 134 & 295 & $T^{1,2}$ & 549 & 217 & 104 & 228 \\
$T^{1, \ldots, 3}$ & 715 & 227 & 201 & 287 & $T^{1, \ldots, 3}$ & 549 & 177 & 157 & 215 \\
$T^{1, \ldots, 4}$ & 715 & 249 & 136 & 330 & $T^{1, \ldots, 4}$ & 549 & 198 & 107 & 244 \\
$T^{1, \ldots, 5}$ & 715 & 233 & 145 & 337 & $T^{1, \ldots, 5}$ & 549 & 194 & 124 & 231 \\
$T^{1, \ldots, 6}$ & 715 & 256 & 124 & 335 & $T^{1, \ldots, 6}$ & 549 & 211 & 102 & 236 \\
$T^{1, \ldots, 7}$ & 715 & 273 & 128 & 314 & $T^{1, \ldots, 7}$ & 549 & 230 & 95 & 224 \\
$T^{1, \ldots, 8}$ & 715 & 283 & 131 & 301 & $T^{1, \ldots, 8}$ & 549 & 251 & 88 & 210 \\
\hline
\end{tabular}

(a) New York

(b) San Francisco

\begin{tabular}{lcccclcccc}
\hline & Total & Inactive & Stable & Active & & Total & Inactive & Stable & Active \\
\hline$T^{1}$ & 481 & & & & $T^{1}$ & 152 & & & \\
$T^{1,2}$ & 481 & 157 & 79 & 245 & $T^{1,2}$ & 152 & 50 & 26 & 76 \\
$T^{1, \ldots, 3}$ & 481 & 100 & 155 & 226 & $T^{1, \ldots, 3}$ & 152 & 37 & 40 & 75 \\
$T^{1, \ldots, 4}$ & 481 & 128 & 88 & 265 & $T^{1, \ldots, 4}$ & 152 & 42 & 30 & 80 \\
$T^{1, \ldots, 5}$ & 481 & 124 & 92 & 265 & $T^{1, \ldots, 5}$ & 152 & 39 & 28 & 85 \\
$T^{1, \ldots, 6}$ & 481 & 146 & 80 & 255 & $T^{1, \ldots, 6}$ & 152 & 46 & 21 & 85 \\
$T^{1, \ldots, 7}$ & 481 & 155 & 89 & 237 & $T^{1, \ldots, 7}$ & 152 & 43 & 27 & 82 \\
$T^{1, \ldots, 8}$ & 481 & 179 & 82 & 220 & $T^{1, \ldots, 8}$ & 152 & 51 & 26 & 75 \\
\hline
\end{tabular}

(c) London

(d) Sydney

\subsection{Loyal Users}

Figure 10 describes the distribution of the percentage of attended events of users among the total events of their groups, and the numbers of attended events of these users in the period of two years. Specifically, Figure 10b,d,f,h demonstrates the numbers of attended events, while Figure 10a,c,e,g illustrates the percentage of the attended events of these users among the total events of their groups. We observe that the majority of users attended fewer than 10 events, as shown in Figure 10b,d,f,h. Moreover, groups of different types created various events; for example, some groups have fewer than 10 events over the two years. That is why the majority of users have a percentage of attended events among all the events of their own groups that is less than $20 \%$, as illustrated in Figure $10 \mathrm{a}, \mathrm{c}, \mathrm{e}, \mathrm{g}$.

In our analysis, our aim is to clarify the relationship between the loyalty of users and the growth of their group over time, so that we evaluate the loyalty of users who attend at least one event in the first time window, $T^{1}$. If a user does not attend any event in $T^{1}$, the user will be removed in the process of evaluation. Otherwise, the user will be selected to compute the loyalty. Hence, to measure the loyalty of a user $u$ in $T^{1, \ldots, i}$, we use Equation (3) to obtain value $\lambda$. We then set threshold $\tau$ to 0.5 , and the user $u$ is loyal within $T^{1, \ldots, i}$, if $\lambda \geqslant \tau$. Otherwise, $u$ is disloyal to group $G$ within $T^{1, \ldots, i}$. 


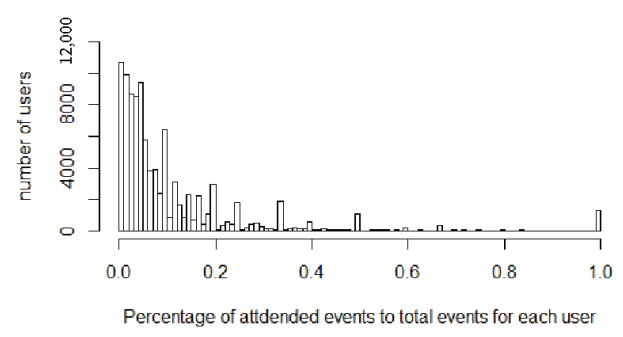

(a)

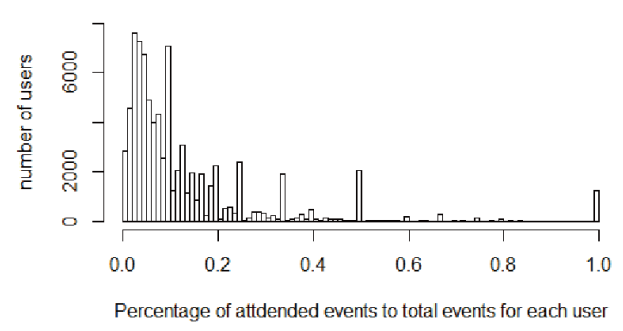

(c)

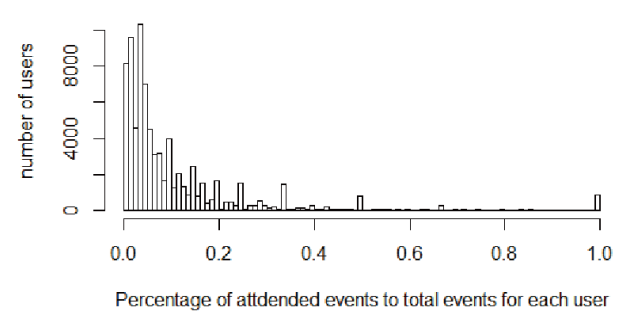

(e)

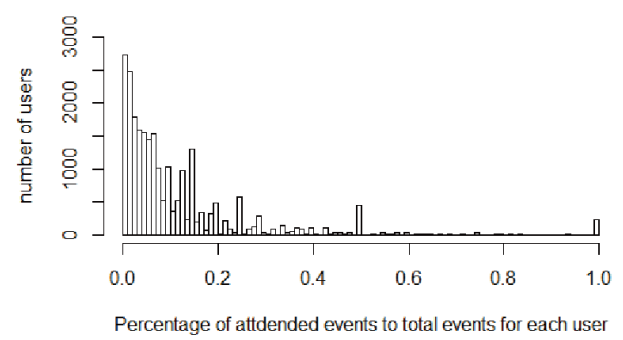

(g)

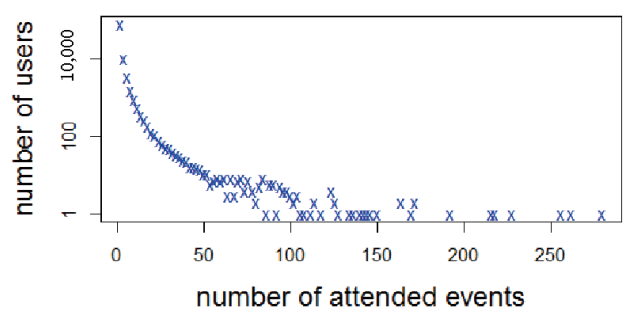

(b)

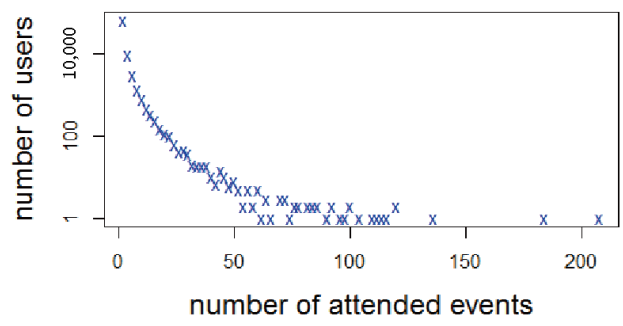

(d)

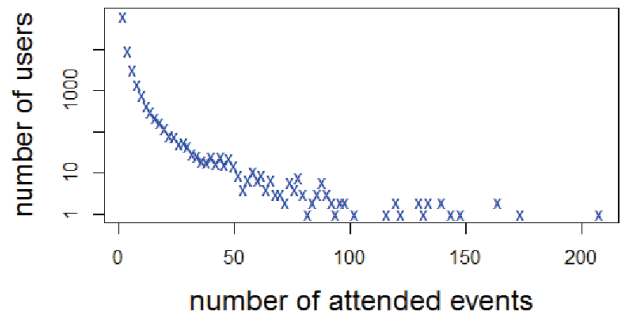

(f)

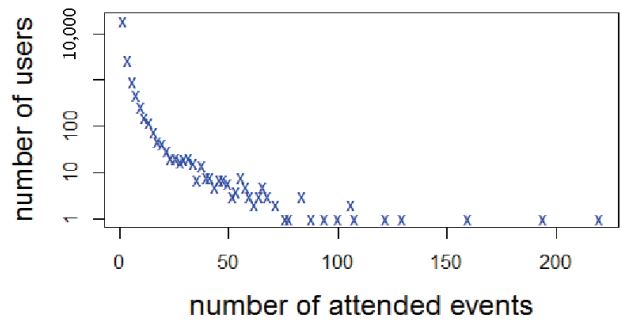

(h)

Figure 10. Distributions in terms of percentage and the number of attended events that users participate in among the total events of their groups in the two-year period. (a) New York-Percentage of attended events in total events for users. (b) New York-Number of attended events for users. (c) San Francisco-Percentage of attended events in total events for users. (d) San Francisco-Number of attended events for users. (e) London-Percentage of attended events in total events for users. (f) London-Number of attended events for users. (g) Sydney-Percentage of attended events in total events for users. (h) Sydney-Number of attended events for users.

Figure 11 illustrates the number of loyal users and the number of disloyal users of all groups in each city. We can observe that the number of users who are loyal to their groups decreases by time. To understand the inactiveness of groups, we investigate the distribution of these loyal users in the 
groups, which is shown in Table 5. Figure 12 describes the distributions of these loyal users in different groups with three activeness labels, i.e., Inactive, Stable, and Active. In general, the numbers of loyal users in groups with Active and those with Stable also decrease. We can observe that a majority of these loyal users attended few events or even left their groups after the first two time windows $T^{1,2}$. Thus, those users leave their group by time due to a loss of interest in their group. Furthermore, in [26], it is argued that few interesting people and low-quality content in the online groups are the main reasons for leaving. Finding people who have the same interests and sharing many things in online groups are very difficult. These are the reasons why few people stay in groups for very long. From our results, we can conclude that a high performance of recommendation systems can be achieved if only a few items are recommended in the systems $[7,8,32]$ within a short time period.

However, there are still some groups that continue to grow. This depends on the group's topic, as well as the members' expansion and relationship, which we will study it in the next article, $A$ New Research Problem of Diffusion Growth in Event-Based Social Networks.

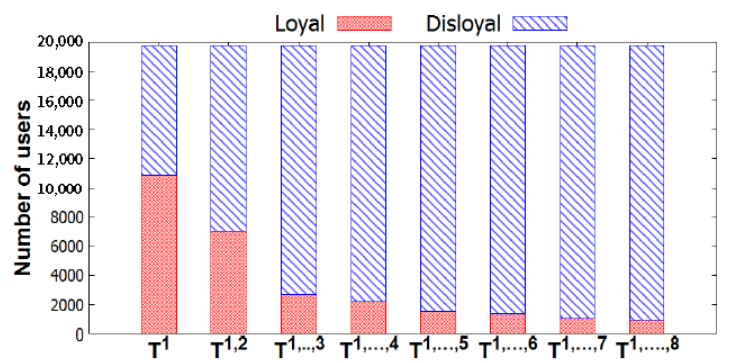

(a)

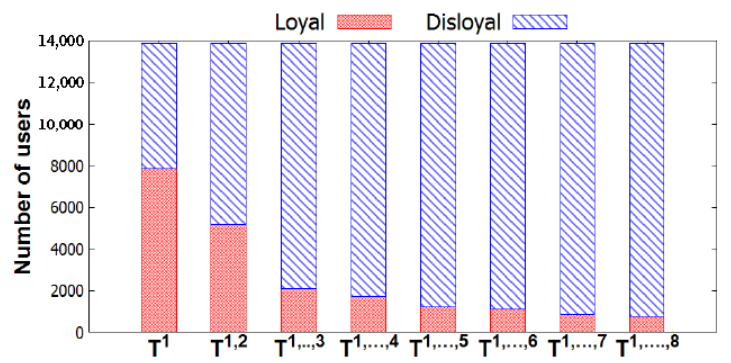

(c)

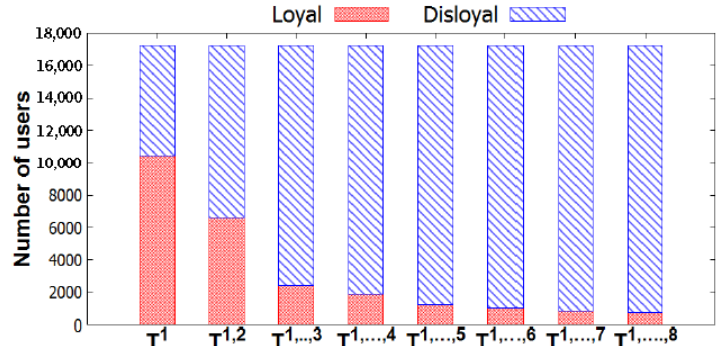

(b)

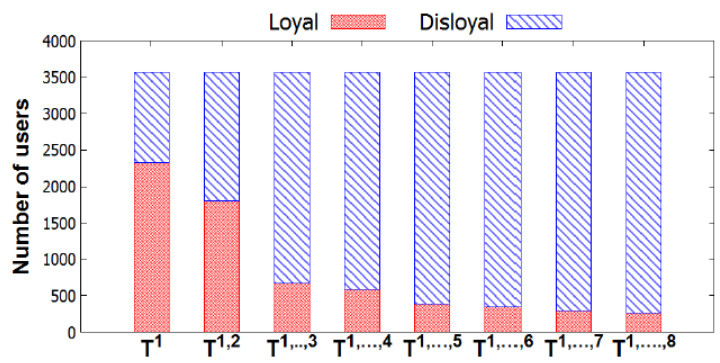

(d)

Figure 11. Numbers of loyal users and disloyal users varying in different $T$ s for the four cities. (a) New York-Number of loyal users and disloyal users. (b) San Francisco-Number of loyal users and disloyal users. (c) London-Number of loyal users and disloyal users. (d) Sydney-Number of loyal users and disloyal users.

\subsection{Activeness Prediction}

We use three well-known classification methods, i.e., RF, decision tree (C5.0), and SVM, to evaluate the results in Table 5. We test these groups in three different series of consecutive time windows, called three stages: $T^{1,2}, T^{1, \ldots, 4}$ and $T^{1, \ldots, 8}$. For each stage in each city, we have a corresponding dataset used in the experiment. Particularly, the dataset of stage $T^{1,2}$ in New York is formed by a set of generated features and activeness labels of groups obtained only in $T^{1,2}$. In the evaluation of this stage, each corresponding dataset was divided randomly into two parts: $70 \%$ for training and $30 \%$ for testing. Each classification method of RF, C5.0, and SVM was used on each dataset 100 times to produce prediction results. The accuracy of each method is obtained by averaging all results of 100 times.

Classical methods, such as Wrapper [16] and Filter [33], are often used to compute the score and remove weak features. However, these methods are not effective for high-dimensional data. Thus, we adopt a method [29] that selects a group of 10 features from high dimensional 
data to result in better accuracy. These feature are listed as N_EVENTS, LOYAL USERS, RSVPS, AVERAGE_Y_RSVPS, SD_Y_RSVPS, AVERAGE_DAY,SD_DAY, N_EVENT_ORGANIZER, N_ORGANIZER, and N_ATTENDEES.

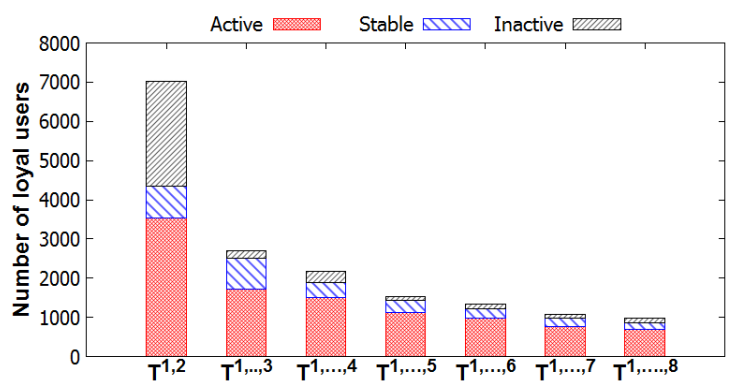

(a) New York

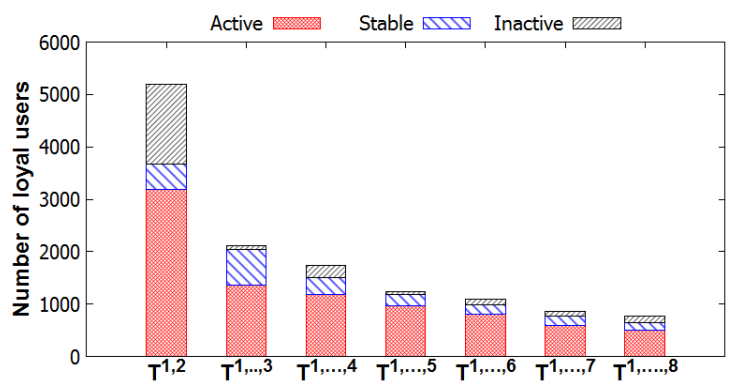

(c) London

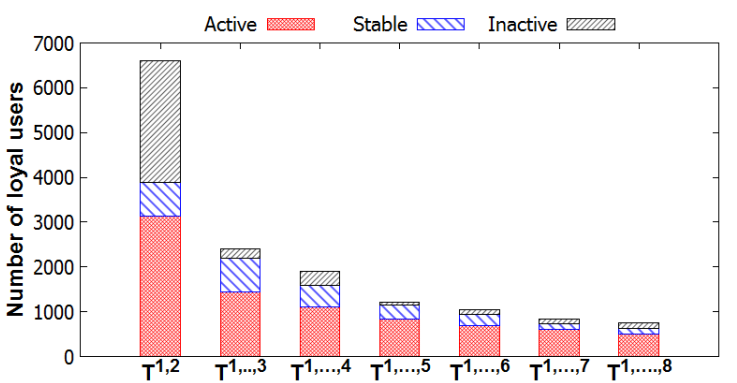

(b) San Francisco

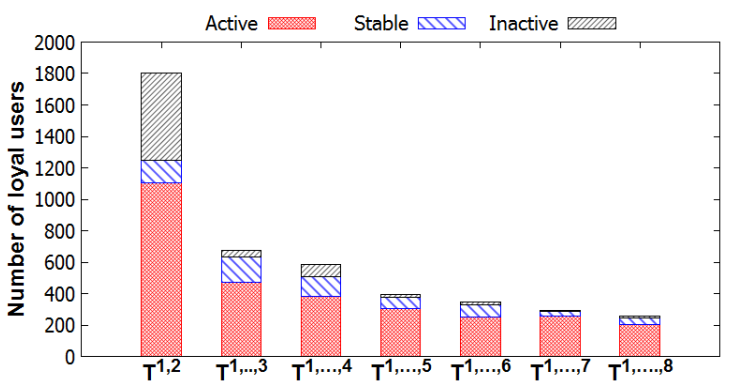

(d) Sydney

Figure 12. Number of loyal users in groups varying in different Ts. Groups are obtained from Table 5.

Table 6 shows the classification results of groups in three activeness classes: active, stable, and inactive. In this table, Column $A L L$ means all features are used in three classifiers for each stage. Column Selected consists of 10 selected features that we used to obtain better results compared with those of $A L L$. We can see that the results predicted by RF are the best, while C5.0 is the worst for both $A L L$ and Selected. Furthermore, the results in Selected are much higher than those in ALL. This indicates that the group of selected features is suitable for all classifiers, and they are strong and highly correlated.

Table 6. Average accuracy of three methods for both $A L L$ and Selected features generated from the three stages.

\begin{tabular}{cccccccc}
\hline & & \multicolumn{3}{c}{ ALL } & \multicolumn{3}{c}{ Selected } \\
\hline & & RF & C50 & SVM & RF & C50 & SVM \\
\hline \multirow{4}{*}{ NY } & $T^{1,2}$ & 69.92 & 65.64 & 69.91 & 71.99 & 68.25 & 71.32 \\
& $T^{1, \ldots, 4}$ & 74.68 & 71.37 & 74.73 & 77.74 & 75.04 & 76.02 \\
& $T^{1, \ldots, 8}$ & 70.64 & 66.19 & 70.21 & 73.07 & 71.37 & 71.58 \\
\hline \multirow{4}{*}{ SF } & $T^{1,2}$ & 69.21 & 66.97 & 69.28 & 72.46 & 69.16 & 70.82 \\
& $T^{1, \ldots, 4}$ & 71.13 & 68.37 & 72.21 & 75.63 & 72.57 & 74.21 \\
& $T^{1, \ldots, 8}$ & 71.47 & 67.69 & 69.72 & 74.59 & 72.94 & 72.15 \\
\hline & $T^{1,2}$ & 69.22 & 61.72 & 71.58 & 70.58 & 65.29 & 73.02 \\
LD & $T^{1, \ldots, 4}$ & 71.5 & 67.61 & 72.15 & 74.66 & 72.51 & 73.82 \\
& $T^{1, \ldots, 8}$ & 69.15 & 62.07 & 68.73 & 70.99 & 67.88 & 69.85 \\
\hline & $T^{1,2}$ & 66.1 & 60.34 & 64.71 & 66.33 & 65 & 68.21 \\
SN & $T^{1, \ldots, 4}$ & 73.04 & 68.8 & 71.41 & 74.84 & 71.52 & 75.15 \\
& $T^{1, \ldots, 8}$ & 69.71 & 60.86 & 66.31 & 68.32 & 64.73 & 71.54 \\
\hline
\end{tabular}




\section{Conclusions}

In this paper, we studied the activeness of groups and the loyalty of users throughout event-based social networks. For this purpose, we used a sliding time window to represent the activity of groups as well as users' participation in each time window. To measure the activeness of groups in several consecutive time windows, we computed the ratio of the numbers of events between two consecutive time windows. We developed an association matrix to assign a suitable activeness label to the group. The loyalty of users was manifested by consistent participation in each time window. To evaluate group activeness labels in a series of consecutive time windows, we used the crawled data of that time to generate features. We then used three well-known classifiers to validate the labels. The study shows that groups that are stable and have thrived have more loyal users. In addition to this, the study also helps to predict how long the loyal users will stay.

Author Contributions: T.T. proposed the research direction, provided conceptualization, crawled the data, implemented the experiment, and wrote the original draft. D.W., J.Z.H., and M.A. analyzed the results and revised the article. All authors contributed equally to the analysis, experiment, and result discussions. All authors have read and agreed to the published version of the manuscript.

Funding: This research received no external funding.

Conflicts of Interest: The authors declare that there is no conflict of interests regarding the publication of this article.

\section{References}

1. Liu, X.; He, Q.; Tian, Y.; Lee, W.C.; McPherson, J.; Han, J. Event-based social networks. In Proceedings of the 18th ACM SIGKDD International Conference on Knowledge Discovery and Data Mining-KDD '12, Beijing, China, 12-16 August 2012; ACM Press: New York, NY, USA, 2012; p. 1032. [CrossRef]

2. Chaudhuri, A.; Holbrook, M.B. The Chain of Effects from Brand Trust and Brand Affect to Brand Performance: The Role of Brand Loyalty. J. Mark. 2001, 65, 81-93. [CrossRef]

3. Hamilton, W.L.; Zhang, J.; Danescu-Niculescu-Mizil, C.; Jurafsky, D.; Leskovec, J. Loyalty in Online Communities. In Proceedings of the Eleventh International AAAI Conference on Web and Social Media (ICWSM 2017), Montréal, QC, Canada, 15-18 May 2017; Volume 2017, pp. 540-543.

4. Palla, G.; Barabási, A.L.; Vicsek, T. Quantifying social group evolution. Nature 2007, 446, 664-667. [CrossRef] [PubMed]

5. Jamali, M.; Haffari, G.; Ester, M. Modeling the temporal dynamics of social rating networks using bidirectional effects of social relations and rating patterns. In Proceedings of the 20th International Conference on World Wide Web, WWW 2011, Hyderabad, India, 28 March-1 April 2011; pp. 527-536. [CrossRef]

6. Nguyen, N.P.; Alim, M.A.; Dinh, T.N.; Thai, M.T. A method to detect communities with stability in social networks. Soc. Netw. Anal. Min. 2014, 4, 1-15. [CrossRef]

7. Jhamb, Y.; Fang, Y. A dual-perspective latent factor model for group-aware social event recommendation. Inf. Process. Manag. 2017, 53, 559-576. [CrossRef]

8. Cao, J., Zhu, Z.; Shi, L.; Liu, B.; Ma, Z. Multi-feature based event recommendation in Event-Based Social Network. Int. J. Comput. Intell. Syst. 2018, 11, 618-633. [CrossRef]

9. Trinh, T.; Nguyen, N.T.; Wu, D.; Huang, J.Z.; Emara, T.Z. A New Location-Based Topic Model for Event Attendees Recommendation. In Proceedings of the 2019 IEEE-RIVF International Conference on Computing and Communication Technologies (RIVF), Danang, Vietnam, 20-22 March 2019; pp. 1-6. [CrossRef]

10. Zhang, J.S.; Lv, Q.I.N. Understanding Event Organization at Scale in Event-Based. ACM Trans. Intell. Syst. Technol. 2019. 10, 1-23. [CrossRef]

11. Patil, A.; Liu, J.; Gao, J. Predicting group stability in online social networks. In Proceedings of theWWW 2013-Proceedings of the 22nd International Conference on World Wide Web, Rio de Janeiro, Brazil, 13-17 May 2013; pp. 1021-1030.

12. Zhang, J.; Tan, L.; Tao, X.; Zheng, X.; Luo, Y.; Lin, J.C.W. SLIND: Identifying Stable Links in Online Social Networks; Lecture Notes in Computer Science; Springer International Publishing: Cham, Switzerland, 2018; Volume 10828, pp. 813-816. [CrossRef] 
13. Zhang, J.; Tao, X.; Tan, L.; Lin, J.C.W.; Li, H.; Chang, L. On Link Stability Detection for Online Social Networks; Springer International Publishing: Cham, Switzerland, 2018; Volume 1, pp. 320-335. [CrossRef]

14. Wu, W.; Antonio, S. Stability Analysis in Dynamic Social Networks Highest Weighted Reward Rule. In Proceedings of the 2010 Spring Simulation Multiconference, Orlando, FL, USA, 11-15 April 2010; pp. 1-6.

15. Ye, M.; Liu, J.; Anderson, B.D.O.; Yu, C.; Basar, T. Evolution of Social Power in Social Networks with Dynamic Topology. IEEE Trans. Autom. Control 2018, 63, 3793-3808. [CrossRef]

16. Kudelka, M.; Horak, Z.; Snasel, V.; Abraham, A. Social network reduction based on stability. In Proceedings of the International Conference on Computational Aspects of Social Networks, CASoN'10, Taiyuan, China, 26-28 September 2010; pp. 509-514. [CrossRef]

17. Liu, J.; Li, Y.; Ling, G.; Li, R.; Zheng, Z. Community Detection in Location-Based Social Networks: An Entropy-Based Approach. In Proceedings of the 2016 IEEE International Conference on Computer and Information Technology (CIT), Nadi, Fiji, 8-10 December 2016; pp. 452-459. [CrossRef]

18. Zhang, Y.; Leezer, J. Emergence of Social Norms in Complex Networks. In Proceedings of the 2009 International Conference on Computational Science and Engineering, Vancouver, BC, Canada, 29-31 August 2009; Volume 4, pp. 549-555. [CrossRef]

19. González-Artega, T.; Cascón, J.M.; de Andrés Calle, R. A proposal to measure human group behaviour stability. In Communications in Computer and Information Science, Proceedings of the 2018 International Conference on Information Processing and Management of Uncertainty in Knowledge-Based Systems, Cádiz, Spain, 11-15 June 2018; Springer: Cham, Switzerland, 2018; Volume 855, pp. 99-110. [CrossRef]

20. Quintane, E.; Pattison, P.E.; Robins, G.L.; Mol, J.M. Short- and long-term stability in organizational networks: Temporal structures of project teams. Soc. Netw. 2013, 35, 528-540. [CrossRef]

21. Tausczik, Y.R.; Dabbish, L.A.; Kraut, R.E. Building Loyalty to Online Communities though Bond and Identity-based Attachment to Sub-groups. In Proceedings of the 17th ACM Conference on Computer Supported Cooperative Work \& Social Computing, Baltimore, MD, USA, 15-19 February 2014.

22. Sharara, H.; Singh, L.; Getoor, L.; Mann, J. Stability vs. Diversity: Understanding the Dynamics of Actors in Time-varying Affiliation Networks. In Proceedings of the 2012 International Conference on Social Informatics, Lausanne, Switzerlan, 14-16 December 2012; pp. 1-6. [CrossRef]

23. Jacoby, J.; Kyner, D.B. Brand Loyalty Vs. Repeat Purchasing Behavior. J. Mark. Res. 1973, 10, 1-9. [CrossRef]

24. Kalwani, M.U.; Narayandas, N. Long-Term Manufacturer-Supplier Relationships: Do They Pay off for Supplier Firms? J. Mark. 1995, 59, 1-16. [CrossRef]

25. Gamboa, A.M.; Gonçalves, H.M. Customer loyalty through social networks: Lessons from Zara on Facebook. Bus. Horizons 2014, 57, 709-717. [CrossRef]

26. Brandtzæg, P.B.; Heim, J. User Loyalty and Online Communities: Why Members of Online Communities are not Faithful. In Proceedings of the 2nd International Conference on INtelligent TEchnologies for Interactive EnterTAINment, Cancun, Mexico, 8-10 January 2008. [CrossRef]

27. Hamilton, W.L.; Zhang, J.; Danescu-Niculescu-Mizil, C.; Jurafsky, D.; Leskovec, J. Loyalty in Online Communities. In Proceedings of ICWSM 2017, Montréal, QC, Canada, 15-18 May 2017.

28. Breiman, L. Random forests. Mach. Learn. 2001, 45, 5-32. [CrossRef]

29. Trinh, T.; Wu, D.; Salloum, S.; Nguyen, T.; Huang, J.Z. A frequency-based gene selection method with random forests for gene data analysis. In Proceedings of the 2016 IEEE RIVF International Conference on Computing and Communication Technologies: Research, Innovation, and Vision for the Future, RIVF 2016, Hanoi, Vietnam, 7 November 2016; pp. 193-198. [CrossRef]

30. Salzberg, S.L. C4.5: Programs for Machine Learning by J. Ross Quinlan. Morgan Kaufmann Publishers, Inc., 1993. Mach. Learn. 1994, 16, 235-240. [CrossRef]

31. Cortes, C.; Vapnik, V. Support-vector networks. Mach. Learn. 1995, 20, 273-297.[CrossRef]

32. Tu, W.; Cheung, D.W.; Mamoulis, N.; Yang, M.; Lu, Z. Activity Recommendation with Partners. ACM Trans. Web 2017, 12, 1-29. [CrossRef]

33. Guyon, I.; Elisseeff, A. An introduction to variable and feature selection. J. Mach. Learn. Res. 2003, 3, 1157-1182.

(C) 2020 by the authors. Licensee MDPI, Basel, Switzerland. This article is an open access article distributed under the terms and conditions of the Creative Commons Attribution (CC BY) license (http://creativecommons.org/licenses/by/4.0/). 